\title{
Tumor Microenvironment-Modulated Nanozymes for NIR-II-Triggered Hyperthermia-Enhanced Photo-Nanocatalytic Therapy via Disrupting ROS Homeostasis
}

Lipeng Zhu'

Yunlu Dai ${ }^{1,2}$

Lizeng $\mathrm{Gao}^{3}$

Qi Zhao (D) ${ }^{1,2}$

'Cancer Centre, Faculty of Health Sciences, University of Macau, Taipa, Macau SAR, People's Republic of China; ${ }^{2}$ MoE Frontiers Science Center for Precision Oncology, University of Macau, Taipa, Macau SAR, People's Republic of China; ${ }^{3} \mathrm{CAS}$ Engineering Laboratory for Nanozyme, Institute of Biophysics, Chinese Academy of Science, Beijing, People's Republic of China
Correspondence: Qi Zhao

Cancer Centre, Faculty of Health

Sciences, University of Macau, Taipa,

Macau SAR, 999078, People's Republic of

China

Email qizhao@um.edu.mo
Purpose: Reactive oxygen species (ROS) are a group of signaling biomolecules that play important roles in the cell cycle. When intracellular ROS homeostasis is disrupted, it can induce cellular necrosis and apoptosis. It is desirable to effectively cascade-amplifying ROS generation and weaken antioxidant defense for disrupting ROS homeostasis in tumor microenvironment (TME), which has been recognized as a novel and ideal antitumor strategy. Multifunctional nanozymes are highly promising agents for ROS-mediated therapy.

Methods: This study constructed a novel theranostic nanoagent based on PEG@ $\mathrm{Cu}_{2-\mathrm{x}}$ $\mathrm{S} @ \mathrm{Ce} 6$ nanozymes (PCCNs) through a facile one-step hydrothermal method. We systematically investigated the photodynamic therapy (PDT)/photothermal therapy (PTT) properties, catalytic therapy (CTT) and glutathione (GSH) depletion activities of PCCNs, antitumor efficacy induced by PCCNs in vitro and in vivo.

Results: PCCNs generate singlet oxygen $\left({ }^{1} \mathrm{O}_{2}\right)$ with laser $(660 \mathrm{~nm})$ irradiation and use catalytic reactions to produce hydroxyl radical $(\bullet \mathrm{OH})$. Moreover, PCCNs show the high photothermal performance under NIR II 1064-nm laser irradiation, which can enhance CTT/ PDT efficiencies to increase ROS generation. The properties of $\mathrm{O}_{2}$ evolution and GSH consumption of PCCNs achieve hypoxia-relieved PDT and destroy cellular antioxidant defense system respectively. The excellent antitumor efficacy in 4T1 tumor-bearing mice of PCCNs is achieved through disrupting ROS homeostasis-involved therapy under the guidance of photothermal/photoacoustic imaging.

Conclusion: Our study provides a proof of concept of "all-in-one" nanozymes to eliminate tumors via disrupting ROS homeostasis.

Keywords: ROS homeostasis, nanozyme, tumor microenvironment, catalytic therapy, photodynamic/photothermal therapy, photothermal/photoacoustic imaging

\section{Introduction}

Reactive oxygen species (ROS), including hydroxyl radical $(\bullet \mathrm{OH})$, superoxide $\left(\cdot \mathrm{O}_{2}{ }^{-}\right)$, and singlet oxygen $\left({ }^{1} \mathrm{O}_{2}\right)$, are a group of signaling biomolecules that play important roles in the cell cycle. ${ }^{1,2}$ Ironically, cancer cells can utilize ROS to drive cell growth, proliferation and signalling pathway for tumor development. ${ }^{3}$ When their concentrations are above a certain level, ROS homeostasis is disrupted by augmenting ROS generation or weakening antioxidant defense, which can induce severe damage to biomolecules and cause cancer cells apoptosis or necrosis. ${ }^{4-6}$ 
Therefore, the disruption of ROS homeostasis through the cascade-amplifying ROS generation and weakening antioxidant defense has been recognized as an effective strategy for anticancer therapy.

Photodynamic therapy (PDT), a most common strategy for generating ROS, utilizes photosensitizers to convert overproduced ${ }^{1} \mathrm{O}_{2}$ under laser illumination. This therapy, a noninvasive cancer treatment, has reduced side effects and high selectivity compared to conventional chemo- and radio-therapies. ${ }^{7-10}$ However, the clinical application of PDT is hindered by low ROS production in the hypoxic tumor microenvironment (TME) and quick energy attenuation. ${ }^{11}$ Therefore, overcoming the limitation of PDT and enhancing the ROS production efficiency is important and imperative to achieve effective antitumor outcomes.

Catalytic therapy (CTT) is another recently developed in situ ROS-enhancement method for antitumor treatment. CTT utilizes iron-initiated Fenton, Fenton-like or nanozyme catalytic reactions to efficiently produce the more cytotoxic ROS $(\cdot \mathrm{OH})$ through the disproportionation of $\mathrm{H}_{2} \mathrm{O}_{2} \cdot{ }^{12-14}$ CTT employs endogenous chemical energy to induce cell death without external energy, avoiding the quick energy attenuation that occurs during penetration of tumor tissue in PDT. ${ }^{15}$ Moreover, CTT is selective, only occurring in tumor tissues instead of normal tissues due to the overproduction of $\mathrm{H}_{2} \mathrm{O}_{2}$ presented in the TME, which makes CTT noninvasive and highly specific therapeutically. ${ }^{16,17}$ Additionally, catalytic reactions concurrently generate $\mathrm{O}_{2}$ in the presence of excess $\mathrm{H}_{2} \mathrm{O}_{2}$ (called catalase-like activity), which relieves the $\mathrm{O}_{2}$-dependent limitations of PDT. ${ }^{18,19}$ Therefore, combining PDT and CTT through using ROS-enhanced nanomaterials can improve the overall treatment of tumors. ${ }^{20,21}$ However, the higher $\mathrm{pH}$ (6.5-6.9) of the TME leads to inefficient catalytic reactions as ferrous-initiated catalytic reactions require a much lower $\mathrm{pH}$ environment (3-5). ${ }^{22}$ The slow reaction rates of ferrous materials has limited their development. ${ }^{23}$ Developing new CTT agents with better catalytic efficiency under neutral physiological conditions is highly desirable.

A large number of metal ion-based nanomaterials (eg, $\mathrm{Mn}, \mathrm{Fe}, \mathrm{Mo}$, and $\mathrm{Cu}$ ) have exhibited great potential for $\mathrm{CTT}$ so far. ${ }^{24-28}$ Among these nanomaterials, Cu-based ones have recently been discovered more adaptable to act as CTT agents because $\mathrm{Cu}^{+}$-based catalytic reactions possess higher catalytic efficiency in neutral and weakly acidic conditions compared with other metal ion-initiated methods. ${ }^{29,30}$ More importantly, $\mathrm{Cu}^{2+}$ catalyzes glutathione (GSH) in the TME to GSSH for accelerating GSH depletion. This destroys cellular antioxidant defense system and regulates the TME to disrupt ROS homeostasis. ${ }^{24,31}$ Additionally, it is confirmed that temperature increases localized to the tumors significantly improve the efficiency of catalytic reactions. ${ }^{32,33}$ Photothermal therapy (PTT) agents convert near-infrared (NIR) light energy to high temperature, ${ }^{34-37}$ augmenting the curative effect and the catalytic efficiency. However, the absorbance of most current PTT agents used for PTT therapy falls within the first NIR I region $(750-1000 \mathrm{~nm})$ whereas the NIR II (1000-1350 nm) region offers deeper tissue penetration and a higher maximum permissible exposure (1.0 $\left.\mathrm{W} \mathrm{cm}{ }^{-2}\right) .{ }^{38,39}$ Therefore, it is highly desired to develop $\mathrm{Cu}-$ based nanozymes that possess NIR-II-excited hyperthermia enhanced CTT/PDT and TME-modulated properties for disrupting ROS homeostasis-mediated therapy.

We first propose this strategy to disrupt ROS homeostasis based on PEG@ $@ \mathrm{Cu}_{2-\mathrm{x}} \mathrm{S} @ \mathrm{Ce} 6$ nanozymes (PCCNs) for cancer theranostics (Figure 1). The PCCNs were synthesized using a facile one-step hydrothermal method with excellent water dispersibility and biocompatibility. The multifunctional PCCNs mediated cascade-amplifying ROS generation and weakening antioxidant defense to disrupt intracellular ROS homeostasis with the following features: (i) the $\mathrm{Ce} 6$ acts as a photosensitizer to generate ${ }^{1} \mathrm{O}_{2}$ under laser $(660 \mathrm{~nm})$ irradiation, (ii) the $\mathrm{Cu}^{+}$in the nanozymes generates more toxic $\cdot \mathrm{OH}$ through catalytic reactions, (iii) the catalase-like property generates $\mathrm{O}_{2}$ simultaneously to enhance the efficiency of hypoxia-relieved PDT, (iv) the redox pair $\left(\mathrm{Cu}^{+} / \mathrm{Cu}^{2}\right.$ ${ }^{+}$) enable GSH depletion through reduction reaction with GSH to disrupt cellular antioxidant defense system and regulate the TME, (v) the PCCNs are hyperthermic PTT agents when exposed to $1064 \mathrm{~nm}$ NIR II laser irradiation and achieve hyperthermia-enhanced CTT and PDT performance. More importantly, the excellent PTT conversion efficiency (44.69\%) of the PCCNs can be applied to photoacoustic/ photothermal imaging. Therefore, PCCNs are a promising "all-in-one" theranostic agent for TME-modulated and cascade-amplifying ROS-mediated PDT/PTT/CTT combination therapy, thus showing superiority over traditional ROS and TME-modulated therapy.

\section{Materials and Methods Materials}

Copper(II) chloride dihydrate $\left(\mathrm{CuCl}_{2} \cdot 2 \mathrm{H}_{2} \mathrm{O}\right)$, SH-5k-PEG, (5,5'-Dithiobis-(2-nitrobenzoic acid)) (DTNB), 2,7-dichlorofluorescin diacetate (DCF), 1, 3-diphenylisobenzofuran (DPBF), 3,3',5,5'-tetramethylbenzidine (TMB) and sodium 


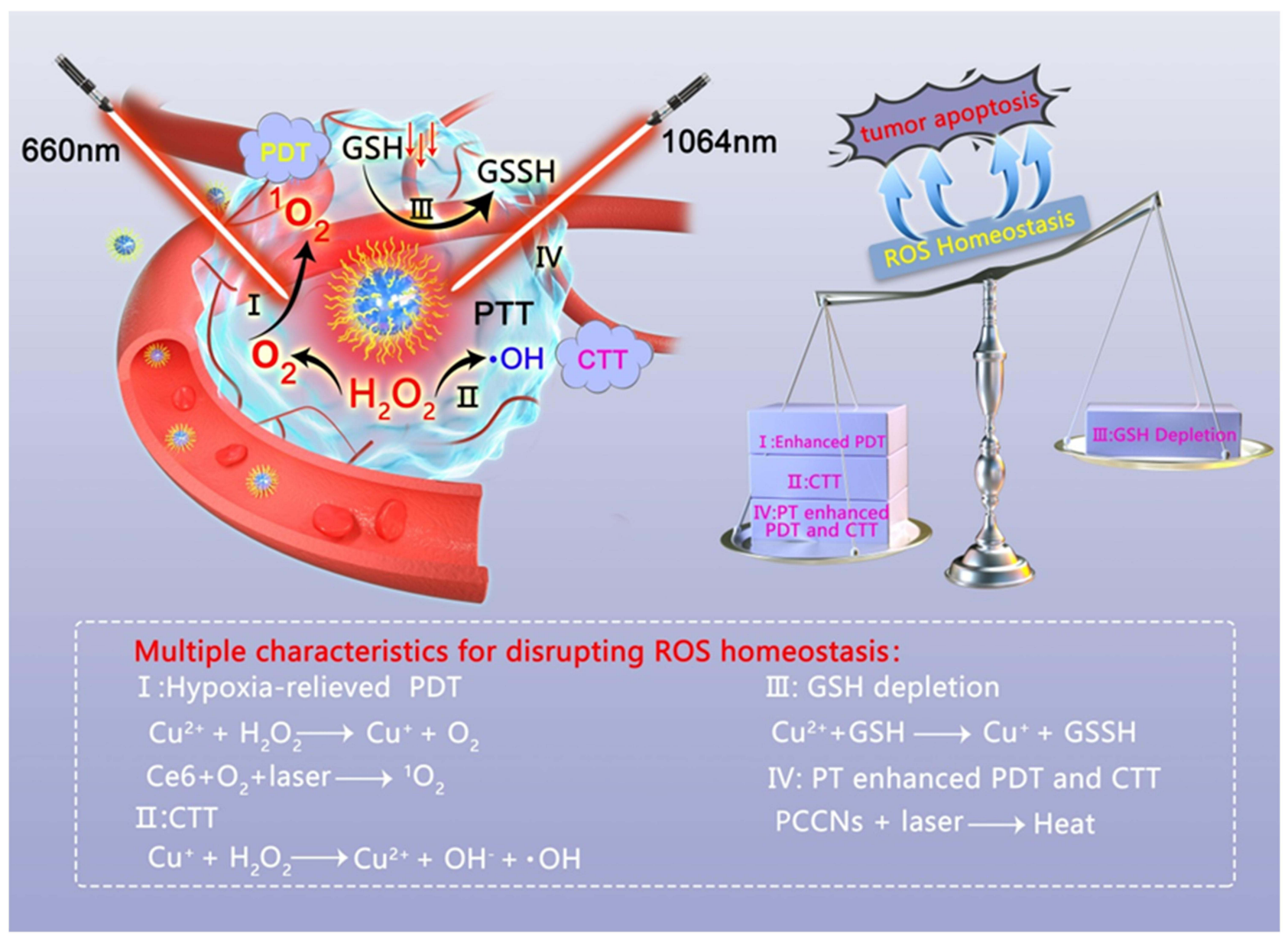

Figure I Schematic illustration of disrupting ROS homeostasis mechanism of PCCNs via the cascade-amplifying ROS generation and weakening antioxidant defense.

hydroxide $(\mathrm{NaOH})$ were purchased from Sigma-Aldrich. Chlorin e6 (Ce6) was acquired from Frontier Scientific. Sodium sulfide nonahydrate $\left(\mathrm{Na}_{2} \mathrm{~S} \cdot 9 \mathrm{H}_{2} \mathrm{O}\right)$ and hydrogen peroxide $\left(\mathrm{H}_{2} \mathrm{O}_{2}\right)$ was obtained from Sinopharm Chemical Reagent Co. Ltd. Calcein-AM/Propidium Iodide (PI) Stained Kit and Cell Counting Kit-8 (CCK-8) were purchased from Shanghai Yeasen Biotechnology Co. Ltd. GSH and GSSG Assay Kit was purchased from Shanghai Beyotime Biotechnology Co. Ltd. All chemicals used in this study were of analytical reagent grade and used without further purification.

\section{Synthesis of the PCCNs}

Ce6 (2 mg mL $\left.{ }^{-1}\right), \mathrm{CuCl}_{2} \cdot 2 \mathrm{H}_{2} \mathrm{O}(0.2 \mathrm{M})$, and PEG were dissolved in water, and then $\mathrm{NaOH}$ was added to adjust the $\mathrm{pH}$ value above 10 . Then, $\mathrm{Na}_{2} \mathrm{~S} \cdot 9 \mathrm{H}_{2} \mathrm{O}(0.2 \mathrm{M})$ was added to the above mixture and heated at $80{ }^{\circ} \mathrm{C}$ for 30 min under protection of $\mathrm{N}_{2}$. Finally, the PCCNs were purified by ultracentrifugation (MWCO 100k) and washed with water several times.

\section{Measurements}

The X-ray diffraction (XRD) patterns were tested with a D8 Focus diffractometer (Bruker). Transmission electron microscopy (TEM) was recorded using an FEI Tecnai G2 S-Twin with a field emission gun operating at $200 \mathrm{kV}$ and equipped with an energy-dispersive X-ray (EDX) spectrometer. The UV-vis absorption spectra were obtained from Lambda 35 spectrophotometer (PerkinElmer). The X-ray photoelectron spectra (XPS) were taken on a VG ESCALAB MK II electron spectrometer using $\mathrm{Mg} \mathrm{K} \alpha(1200 \mathrm{eV})$ as the excitation source. Fourier-transform infrared spectra (FT-IR) were obtained by a Vertex Perkin-Elmer 580BIR spectrophotometer (Bruker). Inductively Coupled Plasma (ICP) was taken on ICAP 6300 of Thermo Scientific. Dynamic light scattering (DLS) and Zeta potential were obtained by using a Malvern instrument Zetasizer Nano system. After 
preparation in water, the sample was lyophilized. Pasting conductive tape on sample stage and taking a few samples on conductive tape for EDS. Nickel grid was used for TEM.

\section{Stability}

To assess the stability of the PCCNs, the size was measured using TEM in different solutions including water, PBS, DMEM and FBS after 7-day treatment.

\section{Photothermal Performance}

The temperature changes of PCCNs at different concentrations were monitored under a $1064-\mathrm{nm}$ laser $\left(0.9 \mathrm{~W} \mathrm{~cm}^{-2}\right.$, $6 \mathrm{~min}$ ) and also monitored under different laser power densities using a thermal imaging camera (Fluke). To evaluate the photostability, the PCCNs were irradiated using a 1064-nm laser $\left(0.9 \mathrm{~W} \mathrm{~cm}^{-2}\right)$ for $6 \mathrm{~min}$ (laser $\mathrm{ON})$, followed by the cooling to room temperature without irradiation for 6 min (laser OFF). Subsequently, the additional three ON/OFF cycles were further repeated.

\section{Photodynamic Properties}

2,7-Dichlorofluorescin diacetate (DCF) was used to detect ROS in PCCNs. The PCCNs were mixed with DCF and followed laser irradiated using a $660-\mathrm{nm}$ laser $\left(20 \mathrm{~W} \mathrm{~cm}^{-2}\right.$, $12 \mathrm{~min}$ ) for different times. The ROS production could be measured by fluorescence of DCF $\left(\lambda_{\text {excitation }}=504 \mathrm{~nm}\right.$ and $\lambda_{\text {emssion }}=529 \mathrm{~nm}$ ). In addition, The ROS production was measured under different temperature and further tested with/without the 1064-nm laser irradiation.

\section{Catalytic Properties}

For the extracellular $\cdot \mathrm{OH}$ generation detection, $\mathrm{PBS}(\mathrm{pH}$ 7.2) containing TMB ( $\left.40 \mu \mathrm{g} \mathrm{mL}^{-1}\right)$ was mixed with PCCNs plus $\mathrm{H}_{2} \mathrm{O}_{2}$. The generation of $\bullet \mathrm{OH}$ was determined by the absorption increase at $650 \mathrm{~nm}$. The TMB solutions treated with PCCNs or $\mathrm{H}_{2} \mathrm{O}_{2}$ alone were used as control groups. In addition, the $\cdot \mathrm{OH}$ generation was measured under different temperatures and further tested with/without the 1064-nm laser irradiation.

Besides, the Michaelis-Menten kinetic assay of the PCCNs was examined by changing the concentration of $\mathrm{H}_{2} \mathrm{O}_{2}(0-3.0 \mathrm{mM})$. Subsequently, the absorbance spectrum at $650 \mathrm{~nm}$ was recorded by UV-Vis absorption spectrophotometer. Finally, the kinetic parameters were obtained according to the previous reported method. ${ }^{40,41}$

\section{Oxygen Generation Activity}

For the $\mathrm{O}_{2}$ production ability of PCCNs, the $\mathrm{H}_{2} \mathrm{O}_{2}$ was added into PCCNs $\left(100 \mu \mathrm{g} \cdot \mathrm{mL}^{-1}\right)$ aqueous solution. Then, the generated concentration of $\mathrm{O}_{2}$ was monitored by a portable dissolved oxygen meter. The PCCNs solutions without $\mathrm{H}_{2} \mathrm{O}_{2}$ treatment were used as control groups.

\section{GSH Depletion Activity}

The depletion of GSH was measured by UV-visible spectroscopy. A DTNB PBS solution $\left(3.0 \mathrm{mg} \cdot \mathrm{mL}^{-1}\right)$ and GSH aqueous solution $(10 \mathrm{mM})$ were added into PCCNs aqueous solution at different concentrations, respectively. After that, the mixtures were maintained at $25{ }^{\circ} \mathrm{C}$ under magnetic stirring for $1 \mathrm{~h}$ and the absorbance of the solution was measured by UV-vis spectroscopy.

\section{Cell Culture}

Mouse mammary carcinoma (4T1) were purchased from the American Type Culture Collection (ATCC). Noncancerous human umbilical vein endothelial cells (HUVECs) were purchased from Thermo Fisher. All cells were cultured in DMEM medium containing $10 \%$ FBS at $37{ }^{\circ} \mathrm{C}$ in a $5 \% \mathrm{CO}_{2}$ humidified atmosphere.

\section{In vitro Cellular Uptake}

The 4T1 cells were seeded on 24-well culture plates $\left(5 \times 10^{4}\right.$ cells/well $)$ and incubated overnight. PCCNs were added into the wells for different time, and then the cells were washed three times using PBS, followed by 3 min incubation with $0.5 \mathrm{~mL}$ of trypsin. Next, the cells were collected through the centrifuge for cell counting, and further lysed under ultrasonication. Finally, the copper ions were extracted using nitric acid from the cells, and measured using ICP-MS.

\section{Cell Viability Assays}

The 4T1 cells and Human umbilical vein endothelial cells (HUVECs) were seeded in 96-well plates at a density of $1 \times 10^{4}$ cells/well and incubated overnight. The PCCNs with different concentrations $(0,7.5,15,30,75,150,225$ $\mu \mathrm{g} \mathrm{mL}^{-1}$ ) was added to each well of the 96-well plate incubated for another $24 \mathrm{~h}$. Then, $10 \mu \mathrm{L}$ of CCK- 8 was added to each well and the plates were incubated at the same condition for an additional $1 \mathrm{~h}$. Finally, the absorbance was measured at $450 \mathrm{~nm}$ by a microplate reader. 


\section{In vitro Synergistic Therapy}

4T1 cells were seeded in 96-well plates at a density of $1 \times 10^{4}$ cells/well overnight. The medium was replaced by the medium containing the PCCNs with or without $\mathrm{H}_{2} \mathrm{O}_{2}$ $(100 \mu \mathrm{M})$. After $4 \mathrm{~h}$, the cells incubated with $\mathrm{H}_{2} \mathrm{O}_{2}$ were irradiated using the $660-\mathrm{nm}$ laser $\left(20 \mathrm{~mW} \mathrm{~cm}^{-2}\right)$ and $1064-$ $\mathrm{nm}$ laser $\left(0.9 \mathrm{~W} \mathrm{~cm}^{-2}\right)$. Then, the cells were further incubated for $4 \mathrm{~h}$ at $37{ }^{\circ} \mathrm{C}$ under $5 \% \mathrm{CO}_{2}$. Finally, the cell viabilities were measured by the CCK- 8 assay.

\section{Live-Dead Cell Staining Experiments}

Calcein-AM/PI co-staining was used to further evaluate the anticancer effects of the PCCNs. 4T1 cells were cultured in 24-well plates for $24 \mathrm{~h}$ and incubated with the PCCNs with or without $\mathrm{H}_{2} \mathrm{O}_{2}(100 \mu \mathrm{M})$ for $4 \mathrm{~h}$. The cells incubated with $\mathrm{H}_{2} \mathrm{O}_{2}$ were irradiated using the $660-\mathrm{nm}$ laser $\left(20 \mathrm{~mW} \mathrm{~cm}^{-2}\right)$ and 1064-nm laser $\left(0.9 \mathrm{~W} \mathrm{~cm}^{-2}\right)$. Then, calcein-AM and propidium iodide (PI) staining reagents were applied to stain the viable cells as green fluorescence $(\lambda \mathrm{ex}=490 \mathrm{~nm}, \lambda \mathrm{em}=515$ $\mathrm{nm})$ and dead cells as red fluorescence $(\lambda \mathrm{ex}=535 \mathrm{~nm}, \lambda \mathrm{em}=$ $617 \mathrm{~nm}$ ). The fluorescence was detected using an inverted fluorescence microscope system (Olympus IX71, JPN).

\section{Intracellular ROS Detection}

The ROS production in vitro was detected by DCF, which is non-fluorescent and could be easily oxidized to green fluorescent DCF by intracellular ROS. 4T1 cells were cultured in 24-well plates for $24 \mathrm{~h}$ and incubated with the PCCNs with or without $\mathrm{H}_{2} \mathrm{O}_{2}$ for $4 \mathrm{~h}$. The cells incubated with $\mathrm{H}_{2} \mathrm{O}_{2}$ were irradiated using the $660-\mathrm{nm}$ laser and 1064-nm laser. Then, the culture media were replaced by fresh culture media, the DCF was added to each well and the mixture was incubated for $20 \mathrm{~min}$. Finally, the cells were washed repeatedly with PBS. The fluorescence was routinely detected by flow cytometry.

\section{Intracellular GSH Measurement}

The Intracellular GSH was measured by the GSH and GSSG Assay Kit (Beyotime). 4T1 cells were cultured in 24-well plates and treated with PBS and PCCNs with different concentrations. After incubating for $6 \mathrm{~h}$, the cells were washed three times with PBS. Then, the cells were collected with centrifugation, and the supernatant was discarded. The cell precipitates were resuspended in protein remover $M$, subjected to three cycles of freezing -thawing, and then centrifugated at $10,000 \mathrm{~g}$ for $10 \mathrm{~min}$ at
$4{ }^{\circ} \mathrm{C}$. The supernatant was reserved for GSH and GSSH assay according to the manufacturer's protocol.

\section{Tumor Model}

The study was conducted according to the guideline of Institutional Animal Care and Use Committee. All animal experimental protocols were approved by the Institutional Animal Care and Use Committee, University of Macau. The approval number was UMARE-041-2020. BALB/c nude mice $(\sim 20 \mathrm{~g})$ were cultured in SPF room, five to a cage. Tumor models were obtained by injecting $2.5 \times 10^{6}$ 4T1 cells into the mice by subcutaneous injection. The tumor size could be used to measure with vernier calipers and then tumor volume was calculated using the following equation: $\mathrm{V}=\left[(\right.$ length $\left.) \times(\text { width })^{2}\right] / 2$.

\section{In vivo Photoacoustic Imaging}

For in vivo photoacoustic imaging (PAI) experiments, the 4T1 tumor-bearing mice were intravenously injected with the PCCNs and the tumor sites were then conducted at 0 , $3,6,12$, and $24 \mathrm{~h}$ after injection using the commercial photoacoustic equipment (Endra Nexus 128, MI). The photoacoustic images were conducted with mice keeping in a water system. The power density of laser on the skin surface of mice was about $9 \mathrm{~mJ} / \mathrm{cm}^{2}$ using a $980 \mathrm{~nm}$ wavelength.

\section{In vivo Photothermal Imaging}

For in vivo photothermal imaging, the tumor-bearing mouse was intravenously injected with the PCCNs $\left(12 \mathrm{mg} \cdot \mathrm{kg}^{-1}\right)$ and PBS. After $6 \mathrm{~h}$, the tumor sites were irradiated using a $1064-\mathrm{nm}$ laser $\left(0.9 \mathrm{~W} \mathrm{~cm}^{-2}\right)$ for $6 \mathrm{~min}$. During the NIR irradiation process, the infrared thermal camera was used to monitor the temperature changes of the tumor sites.

\section{In vivo Biodistribution}

Tumor-bearing mice were injected with the PCCNs. The main organs of the mice, such as heart, liver, spleen, lung, kidney and tumor, were collected at different time points. Then all of the organs were treated with concentrated nitric acid and $\mathrm{H}_{2} \mathrm{O}_{2}(\mathrm{v} / \mathrm{v}=1: 2)$ on heating $\left(70{ }^{\circ} \mathrm{C}\right)$ until the solutions became clear. The concentrations of copper in the solutions were measured by ICP-MS, and the concentrations in each organ were calculated. 


\section{In vivo Synergistic Therapy}

To evaluate the efficiency of chemodynamic/photodynamic/photothermal synergistic therapy, tumor-bearing mice were randomly divided into five groups: 1) PBS, 2) PCCNs (CTT group), 3) PCCNs plus 660-nm laser (CTT/ PDT group), 4) PCCNs plus 1064-nm laser (CTT/PTT group), and 5) PCCNs plus 660-nm and 1064-nm laser (CTT/PDT/PTT group). Mice were intravenously injected with the PCCNs $\left(12 \mathrm{mg} \cdot \mathrm{kg}^{-1}\right)$. At $6 \mathrm{~h}$ post-injection, the tumors (three and five groups) were irradiated with a 660$\mathrm{nm}$ laser $\left(20 \mathrm{~mW} \mathrm{~cm}^{-2}\right)$ for $20 \mathrm{~min}$, and the tumors (four and five groups) were exposed to an 1064-nm laser (0.9 $\mathrm{W} \mathrm{cm}{ }^{-2}$ ) for $6 \mathrm{~min}$. The mice weight and tumor volume were monitored every 2 days. According to the animal protocol, tumor volume reaching $1500 \mathrm{~mm}^{3}$ were euthanized. Additionally, the tumor was obtained and sectioned into $10-\mu \mathrm{m}$ slices to stain by hematoxylin and eosin (H\&E), HIF-1 $\alpha$, and TUNEL staining.

\section{In vivo Biocompatibility Evaluation}

Tumor-bearing mice were intravenously injected with the PCCNs and PBS. Mice were euthanized after therapy and main organs were obtained, and fixed with paraformaldehyde $(4 \%)$ and stained by $H \& E$ before inverted microscope observation. Meanwhile, serum levels of aspartate aminotransferase (AST) and alanine aminotransferase (ALT) were measured after post-injection by commercial kits.

\section{Statistical Analysis}

Statistical analysis was conducted by the Student's $t$-test, and differences were set at $\mathrm{p}<0.05\left({ }^{*} \mathrm{p}<0.05,{ }^{* *} \mathrm{p}<0.01\right.$, $* * * \mathrm{p}<0.001)$.

\section{Results}

\section{Synthesis and Characterization of PCCNs}

We firstly synthesized the multifunctional PCCNs using a facile one-step hydrothermal method. The PCCNs were successfully synthetized in aqueous solution within $30 \mathrm{~min}$ through the reaction of $\mathrm{CuCl}_{2}$ and $\mathrm{Na}_{2} \mathrm{~S}$ in the presence of Ce6 and PEG-SH (Figure 2A). Because of strong conjugation of $\mathrm{Cu}$ ions with carboxyl groups of $\mathrm{Ce} 6$ and thiol groups of PEG-SH, Ce6 and PEG-SH were not only utilized to be the capping agent for stabilizing PCCNs but also used as template for homogeneous growth of PCCNs to obtain uniform distribution of particle size. Unlike previous methods, ${ }^{42}$ we created a rapid one-step strategy and user- friendly synthesis process that did not require toxic organic solvents and raw materials. Most importantly, PCCNs could be acted as CTT/PDT/PTT, and TME-modulated agents without further surface modification. Transmission electron microscope (TEM) images presented that the as-synthesized PCCNs displayed good monodispersibility and uniform spherical morphology (Figure 2B). The PCCNs were ultrasmall with an average diameter of $5.18 \mathrm{~nm}$ (Figure 2B, $\underline{\mathrm{S} 1}$ ). The lattice spacing in the high-resolution TEM (HRTEM) images was about $0.23 \mathrm{~nm}$ and $0.25 \mathrm{~nm}$ (Figure 2C). The PCCNs could be well dispersed in water with a mean hydrodynamic diameter of $\sim 32.67 \mathrm{~nm}$ in Figure S2, which was larger than the size measured by TEM because of the hydration effect. As shown in Figure 2D, there were basically diffraction peaks for PCCNs at $23.2^{\circ}, 26.8^{\circ}, 30.6^{\circ}$, $36.1^{\circ}, 39.4^{\circ}, 42.9^{\circ}, 45.2^{\circ}$, and $48.7^{\circ}$. These peaks were related to lattice planes of (100), (103), (105), (106), (008), and (110) as the covellite CuS phase (JCPDS file: 06-0464). ${ }^{43-45}$ The results were consistent with a selected area electron diffraction (SAED) pattern $(103,105$, and 008) (Figure S3). The analysis of EDS confirmed that $\mathrm{S}$ and $\mathrm{Cu}$ had a coinciding distribution and the molar ratio of $\mathrm{S} / \mathrm{Cu}$ obtained from quantitative analysis was $\sim 2: 3$ (Figure 2E). The spectra of the X-ray photoelectron spectroscopy (XPS) presented the peaks of 952.5/953.45 eV and 932.75/933.7 eV respectively in accordance with the $\mathrm{Cu}$ $2 \mathrm{p} 1 / 2$ and $\mathrm{Cu} 2 \mathrm{p} 3 / 2$ levels (Figure S4), verifying the coexistence of $\mathrm{Cu}^{+} / \mathrm{Cu}^{2+}$. From the XPS spectra of PCCNs (Figure 2F), two $\mathrm{O} 1 \mathrm{~s}$ peaks at 530.6 and $532.4 \mathrm{eV}$ were assigned to $\mathrm{C}=\mathrm{O}$ from $\mathrm{Ce} 6$ and $\mathrm{C}-\mathrm{O}-\mathrm{C}$ from $\mathrm{PEG}$, respectively. As shown in Figure 2G, the PCCNs showed several same peaks with Ce6 and PEG in the FT-IR spectra (Table

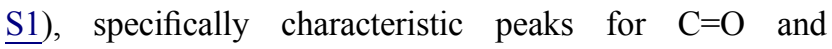
C-O-C. These results indicated the existence of PEG and $\mathrm{Ce} 6$ in the nanozymes. UV-vis spectra showed that PCCNs displayed the same characteristic peak of $645 \mathrm{~nm}$ and 401 nm with Ce6 and also led to a significant blue shift, probably due to the strong interaction between Ce6 and nanozymes (Figure 2H). As shown in the TEM images (Figure 2I), no obvious size changes of PCCNs in water, PBS, DMEM, and FBS for 7 days after treatment. As stated above, the PCCNs maintained good stability in physiological media and would be suitable for further biological applications.

\section{The Photothermal Properties of PCCNs}

UV-vis spectra showed that the PCCNs exhibited the strong NIR II absorption (Figure S5), which allowed their great 
A

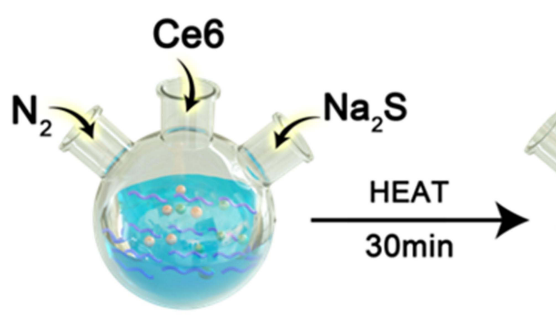

C

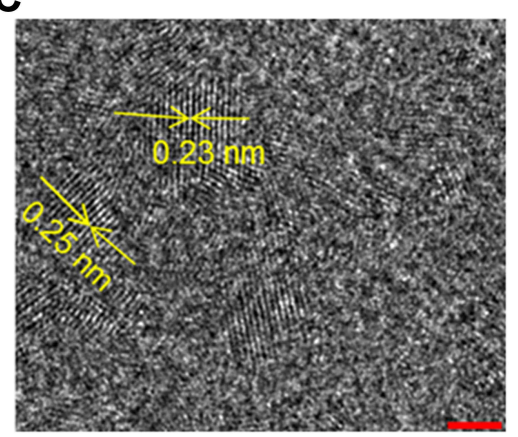

F

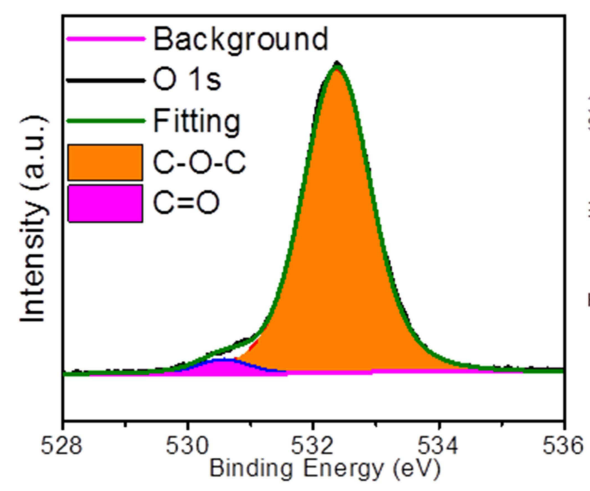

I

Water

\section{D}

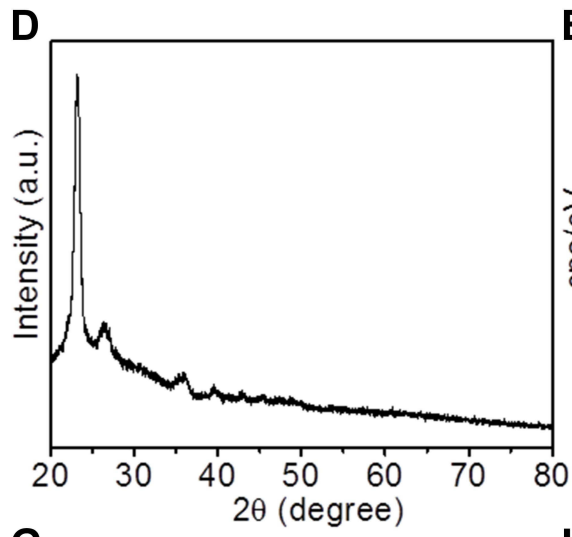

G

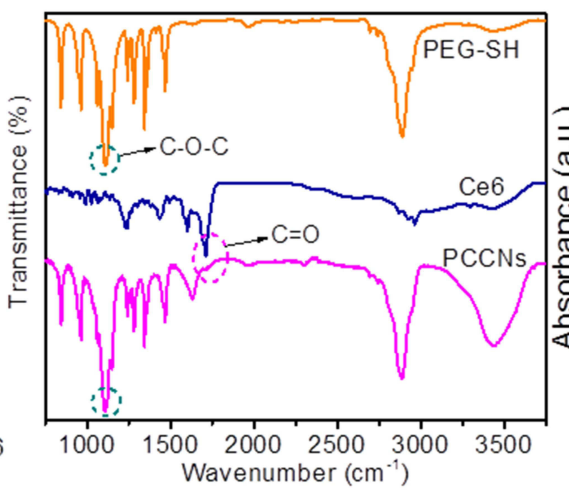

B

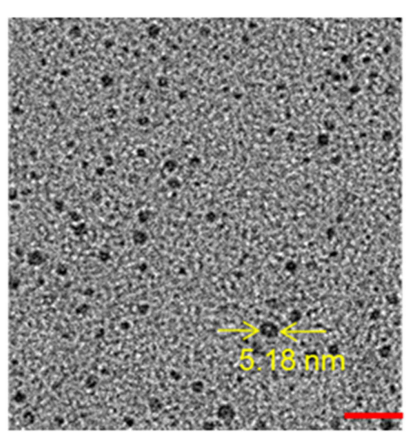

E

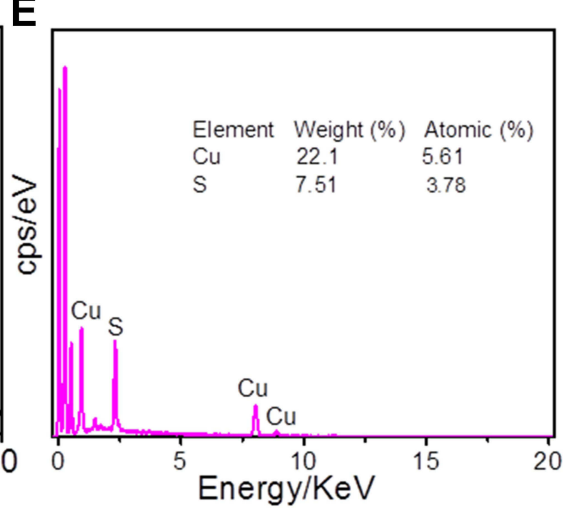

H

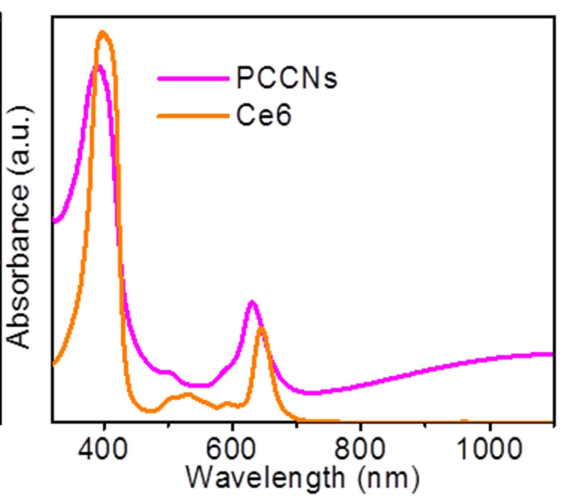

PBS

DMEM

FBS
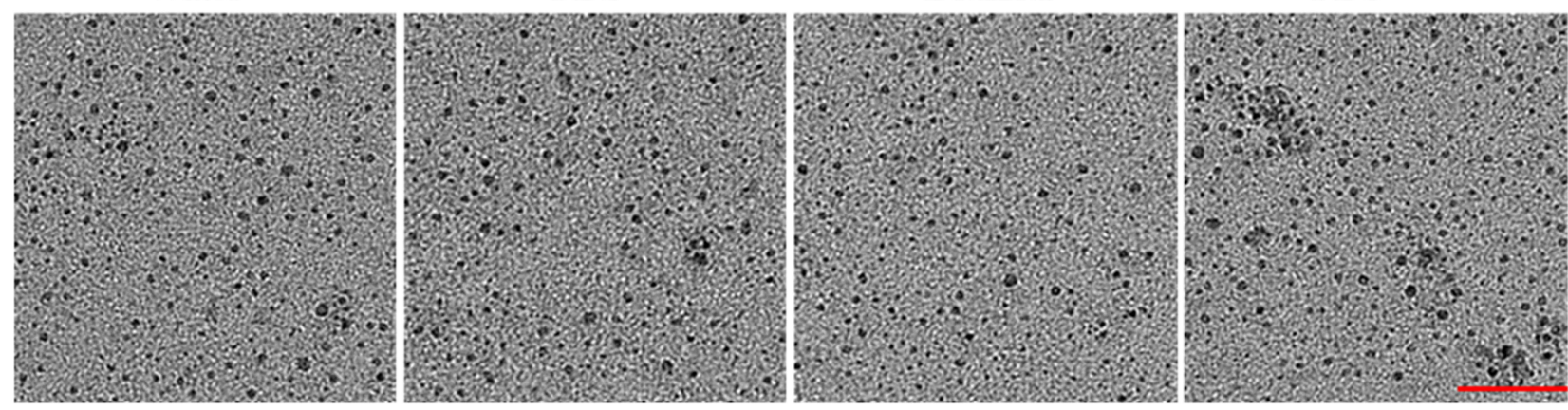

Figure 2 Synthesis and Characterization of PCCNs. (A) Schematic illustration of the synthesis of multifunctional PCCNs. (B) TEM images of PCCNs. Scale bar: $20 \mathrm{~nm}$. (C) HR-TEM images of PCCNs. Scale bar: $2.5 \mathrm{~nm}$. (D) XRD patterns of PCCNs. (E) EDS characterization of PCCNs. (F) High resolution O Is in PCCNs. (G) FT-IR spectra of Ce6, PEG-SH, and PCCNs. (H) UV-vis spectrum of Ce6 and PCCNs. (I) TEM of PCCNs dispersed in water, PBS, DMEM, and FBS for 7 days treatment. Scale bar: $50 \mathrm{~nm}$. 
potential in PTT performance under laser irradiation. As shown in Figure $3 \mathrm{~A}$ and $\mathrm{B}$, the temperature of PCCNs markedly increased to $60.8^{\circ} \mathrm{C}$ under 1064 -nm laser irradiation $\left(0.9 \mathrm{~W} \mathrm{~cm}^{-2}, 6 \mathrm{~min}\right)$. In sharp contrast, the temperature of PBS was only raised by $6.6^{\circ} \mathrm{C}$. As shown in Figure 3B, with the temperature rising from $38.6^{\circ} \mathrm{C}$ to $60.8^{\circ} \mathrm{C}$ in the concentration range of $25-200 \mu \mathrm{g} \mathrm{mL}^{-1}$, PCCNs displayed the concentration-dependent photothermal effect. The temperatures increased from $41.3^{\circ} \mathrm{C}$ to $69^{\circ} \mathrm{C}$ in power densities range of $0.6-1.5 \mathrm{~W} \mathrm{~cm}^{-2}$ (Figure $3 \mathrm{C}$ ), suggesting laser power density-dependent photothermal effect. According to the reported method (Figure S6 and the calculation of the photothermal conversion efficiency section in the Supplementary materials), the PTT conversion efficiency was calculated to be $44.69 \%$, which was higher than many $\mathrm{CuS}$ nanomaterials, such as PAA-CuS NCs (19.5\%), $\mathrm{CuS} @ \mathrm{CP}$ (34\%), GSH-CuS NDs (21.9\%), and $\mathrm{Cu}_{9} \mathrm{~S}_{5}$ $(25.7 \%){ }^{46-49}$ Furthermore, no significant differences were observed in the temperature changes after four cycles of irradiation, demonstrating the excellent photostability of the PCCNs (Figure 3D). Therefore, the excellent PTT property and outstanding photostability of PCCNs allow these PCCNs to achieve efficient PTT effects in cancer treatment.

\section{The Photodynamic Properties of PCCNs}

Next, the photodynamic property of PCCNs was evaluated using the ROS sensor 2',7'-dichlorofluorescein diacetate (DCF). As shown in Figure 4A, the fluorescence signal of ROS obviously increased gradually with increasing irradiation time when the PCCNs were exposed to the 660-nm laser irradiation. These results certified that the ROS generation ability would not be affected by the conjugation of $\mathrm{Ce} 6$ to PCCNs, suggesting their great potential for PDT applications. As shown in Figure 4B, no obvious DCF fluorescence signal was detected for PBS and DCF, indicating that they cannot produce ROS. Compared with that under only 660-nm laser
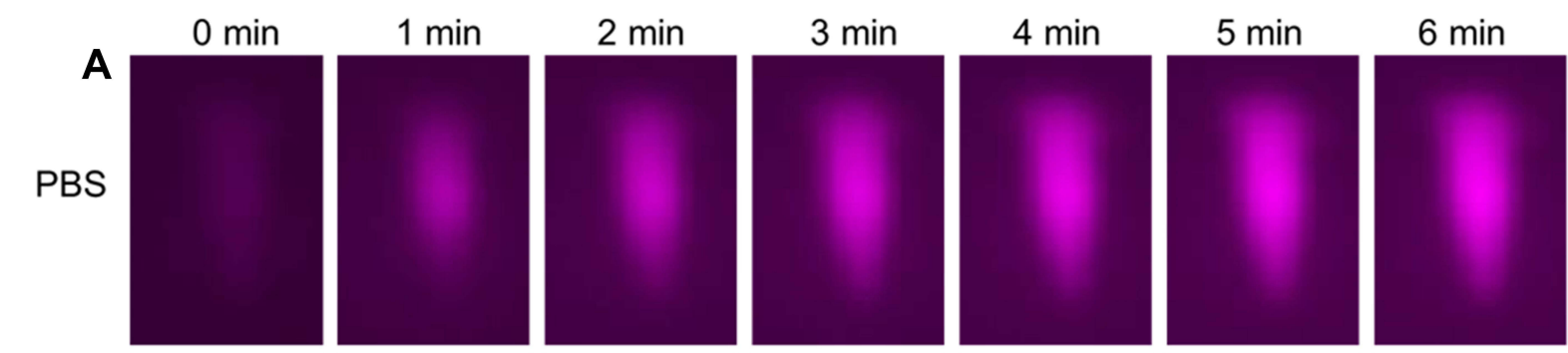

$92^{\circ} \mathrm{C}$
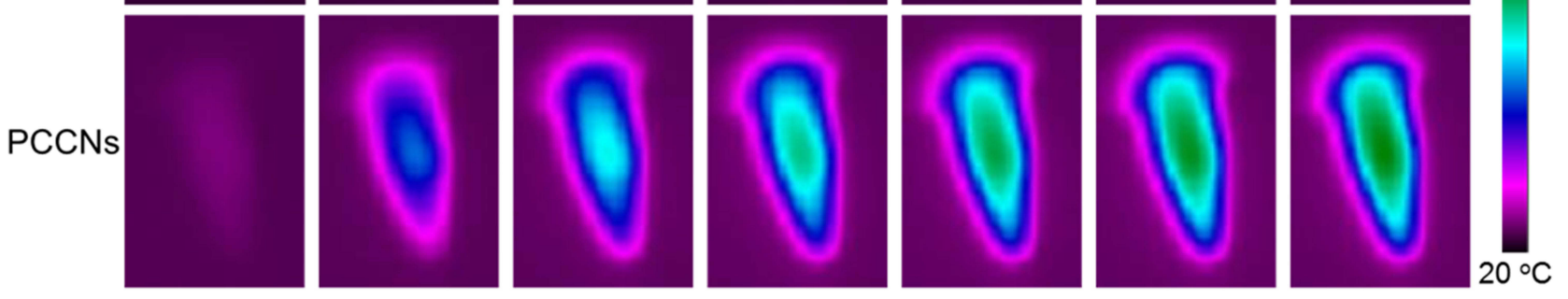

B

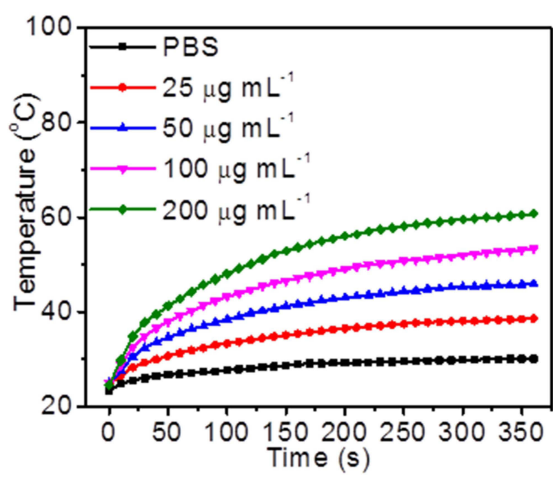

C

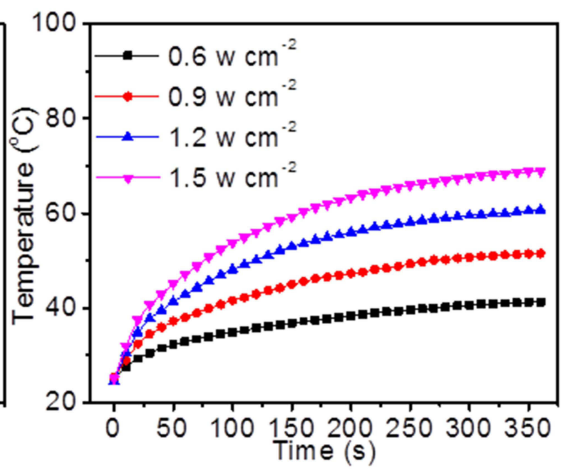

D

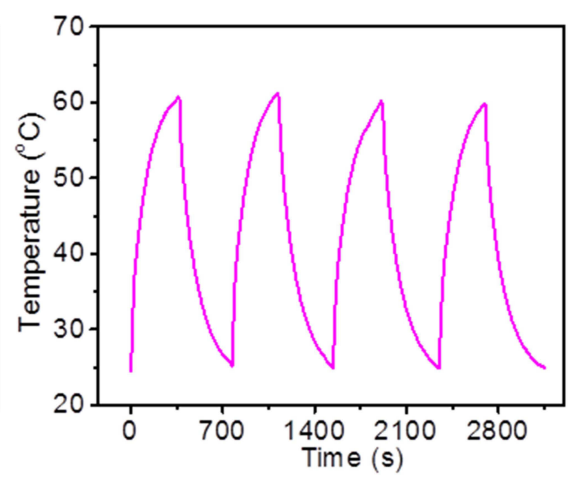

Figure 3 (A) The thermographic images of PBS and PCCNs at different intervals under I064-nm laser $\left(0.9 \mathrm{~W} \mathrm{~cm}^{-2}\right)$ irradiation. (B) The temperature elevation curves of PCCNs under different concentrations. (C) The temperature elevation curves of PCCNs under different laser power intensities. (D) The temperature variations of PCCNs over four cycles of heating and natural cooling. 

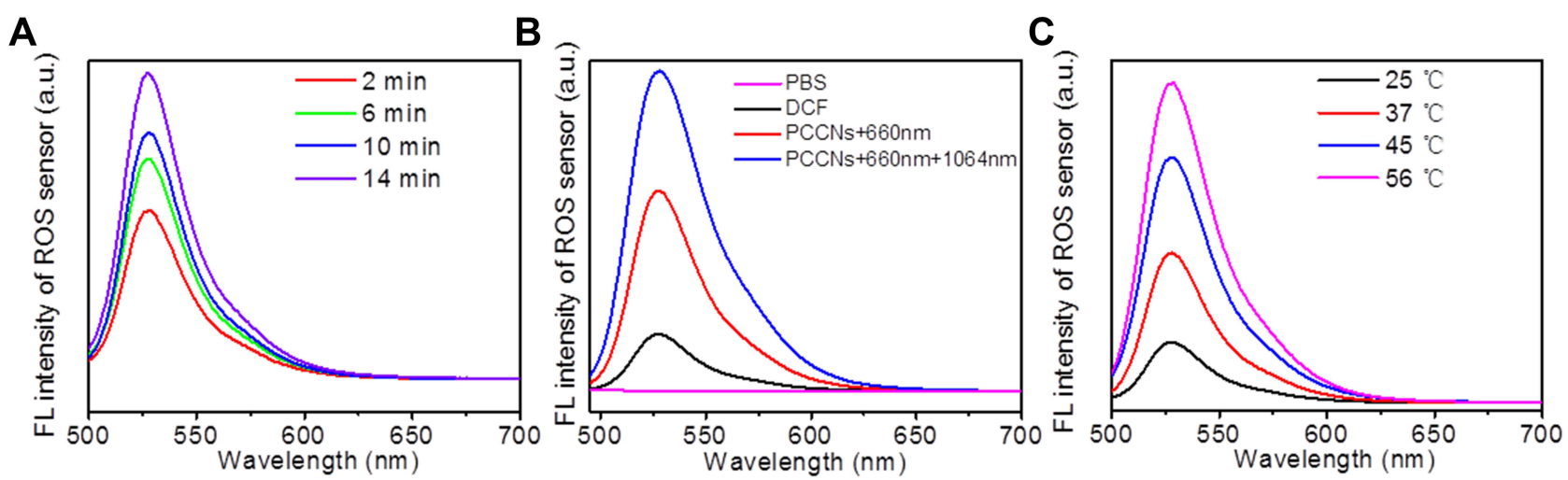

Figure 4 (A) The fluorescence intensity of DCF in PCCNs using a 660-nm laser under different irradiation time. (B) The fluorescence intensity of DCF from different groups. (C) The FL intensity of DCF in PCCNs using a 660-nm laser under different temperatures.

irradiation, the DCF fluorescence signal of PCCNs was significantly enhanced under the simultaneous laser irradiation at $660 \mathrm{~nm}$ and $1064 \mathrm{~nm}$, which showed that PTT could enhance ROS generation. The relationship between higher ROS generation and the increment of temperature $\left(25^{\circ} \mathrm{C}\right.$ to $\left.56^{\circ} \mathrm{C}\right)$ was confirmed (Figure 4C). Collectively, these results showed that PCCNs have a great potential for PTT-enhanced PDT.

\section{$\mathrm{O}_{2}$-Producted and GSH-Depleted Properties of PCCNs}

To evaluate the catalase-like activities of PCCNs, the changes of $\mathrm{O}_{2}$ contents were monitored using a dissolved oxygen meter. Compared with that for PCCNs, an increment in the $\mathrm{O}_{2}$ level was observed after PCCNs and $\mathrm{H}_{2} \mathrm{O}_{2}$ treatment (Figure 5A). These results demonstrated that the PCCNs had catalase-like activities to produce $\mathrm{O}_{2}$, which indicated that PCCNs could achieve the hypoxia-relieved PDT effect. Additionally, the GSH depletion properties of PCCNs were investigated using the GSH kit. As shown in Figure 5B, the content of GSH was decreased with the increase concentrations in PCCNs because the $\mathrm{Cu}^{+} / \mathrm{Cu}^{2+}$ redox pair in the nanozymes provided great potential for redox reaction with GSH. Therefore, GSH consumption could effectively impair tumor antioxidant defense system in the TME for significantly disrupting intracellular ROS homeostasis.

\section{Catalytic Properties of PCCNs}

To further investigate catalytic properties, the $\bullet \mathrm{OH}$ generation of PCCNs was determined using 3,3',5,5'tetramethylbenzidine (TMB) assay, based on the fact that TMB can be oxidized by the highly reactive $\cdot \mathrm{OH}$ to give a blue-green color with the maximum absorbance at about $650 \mathrm{~nm}$. No significant increase of TMB absorption was found in $\mathrm{H}_{2} \mathrm{O}_{2}$ or PCCNs compared to PCCNs plus $\mathrm{H}_{2} \mathrm{O}_{2}$ (Figure 6A). These results demonstrated the occurrence of the catalytic reactions between $\mathrm{PCCNs}$ and $\mathrm{H}_{2} \mathrm{O}_{2}$. As shown
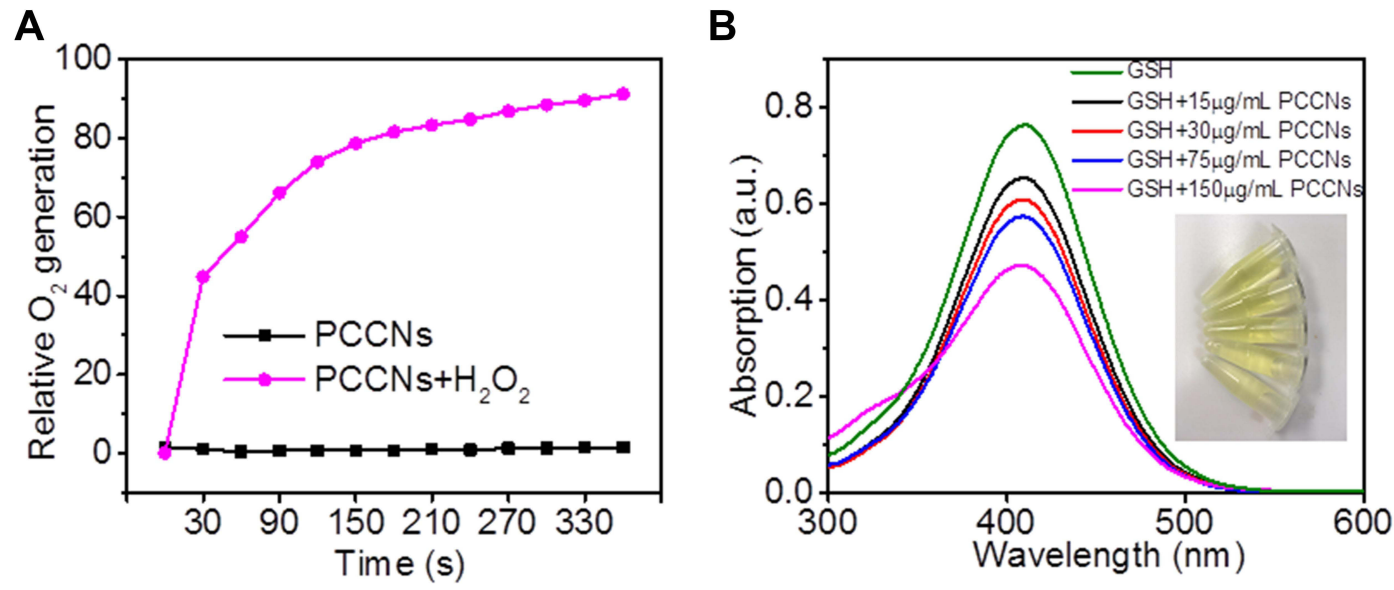

Figure 5 (A) $\mathrm{O}_{2}$ generation of $\mathrm{PCCNs}$ with $\mathrm{H}_{2} \mathrm{O}_{2}$. (B) GSH depletion under different concentrations of PCCNs. 

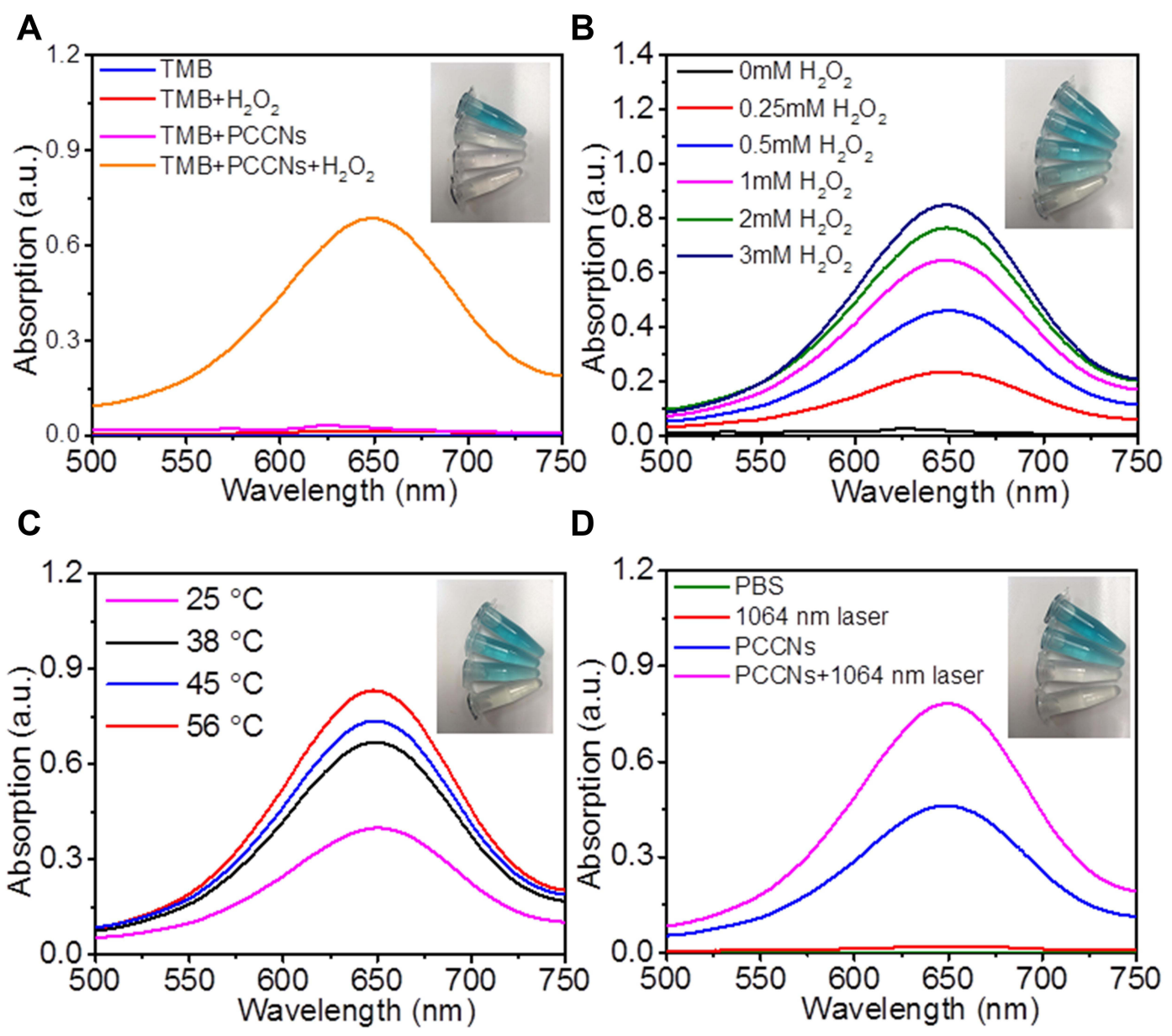

Figure 6 (A) UV-vis spectra and photographs (inset) of TMB aqueous solution from different groups. (B) UV-vis spectra and photographs (inset) of TMB in PCCNs treated with different $\mathrm{H}_{2} \mathrm{O}_{2}$ concentrations. (C) UV-vis spectra and photographs (inset) of TMB aqueous solution from different temperatures. (D) UV-vis spectra and photographs (inset) of TMB in PCCNs solution treated with/without 1064 laser irradiation.

in Figure 6B, the absorption peak of TMB in the PCCNs treatment gradually increased with increasing of $\mathrm{H}_{2} \mathrm{O}_{2}$, which suggested that PCCNs could catalyse the oxidation of TMB in the presence of $\mathrm{H}_{2} \mathrm{O}_{2}$ in a concentration-dependent manner. As shown in Figure S7, the typical Michaelis-Menten kinetics in the concentration range of $\mathrm{H}_{2} \mathrm{O}_{2}$ was exhibited with a Michaelis-Menten constant $\left(\mathrm{K}_{\mathrm{m}}\right)$ and maximal reaction velocity $\left(V_{\max }\right)$ (Table S2), suggesting the effective catalytic activity of PCCNs. Moreover, the temperature increase also induced $\bullet \mathrm{OH}$ generation (Figure $6 \mathrm{C}$ ), indicating that the temperature enhances the catalytic rate of the catalytic reactions. Increased $\bullet \mathrm{OH}$ generation by PCCNs under the 1064-nm laser irradiation was confirmed (Figure 6D), which revealed that PTT could enhance catalytic efficiency. The whole experiment was carried out under neutral $\mathrm{pH}$ of 7.2 , consistent with previous observation, which was different from that of the ferrous-based Fenton reaction. ${ }^{23}$ This property showed that PCCNs could display a strong catalytic rate under the neutral conditions thus making them excellent CTT agents against cancer. Taken together, these results confirmed that PCCNs could act as "all in one" theranostic agents for cascadeamplifying ROS generation and weakening antioxidant defense in the TME for significantly disrupting ROS homeostasis to favor ROS-mediated therapy.

\section{In vitro Cellular Uptake}

The excellent therapeutic efficacy was mainly associated with cellular uptake, which was measured by inductively coupled plasma mass spectrometry (ICP-MS). As shown in Figure 7A, an obvious increase of $\mathrm{Cu}$ amount was observed in 4T1 cells incubated for $24 \mathrm{~h}$ compared with that in cells incubated for $6 \mathrm{~h}$. This suggested that cellular uptake of PCCNs gradually increased with time increasing. The $\mathrm{Cu}$ amount in 4T1 cells increased with the increasing of PCCNs, so the concentration-dependent cellular uptake efficacy of PCCNs was confirmed. 


\section{A}
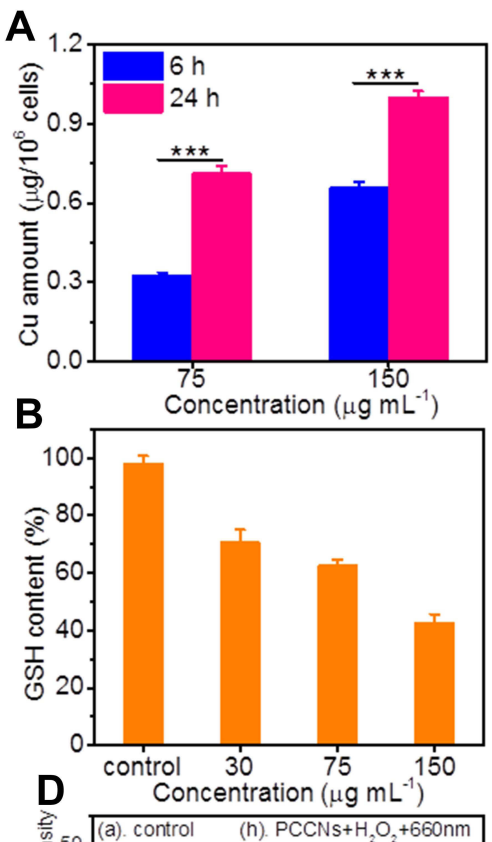

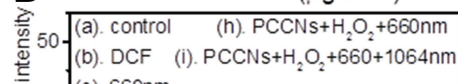

Dू
(c) 40

(d). (eCCNs

30 (f). $\mathrm{H}_{2} \mathrm{O}_{2}$

学 $(\mathrm{g})$. $\mathrm{PCC}$

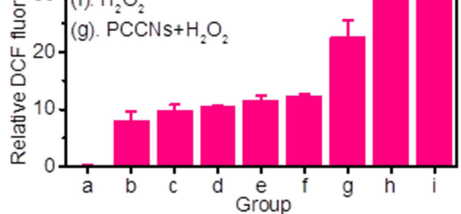

C

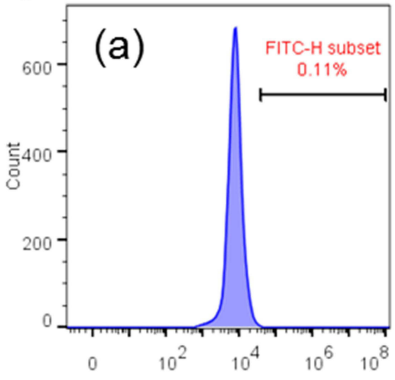

$(\mathrm{d})$
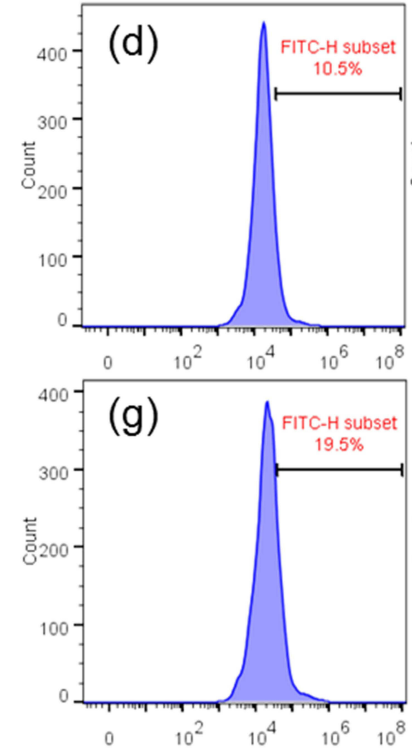
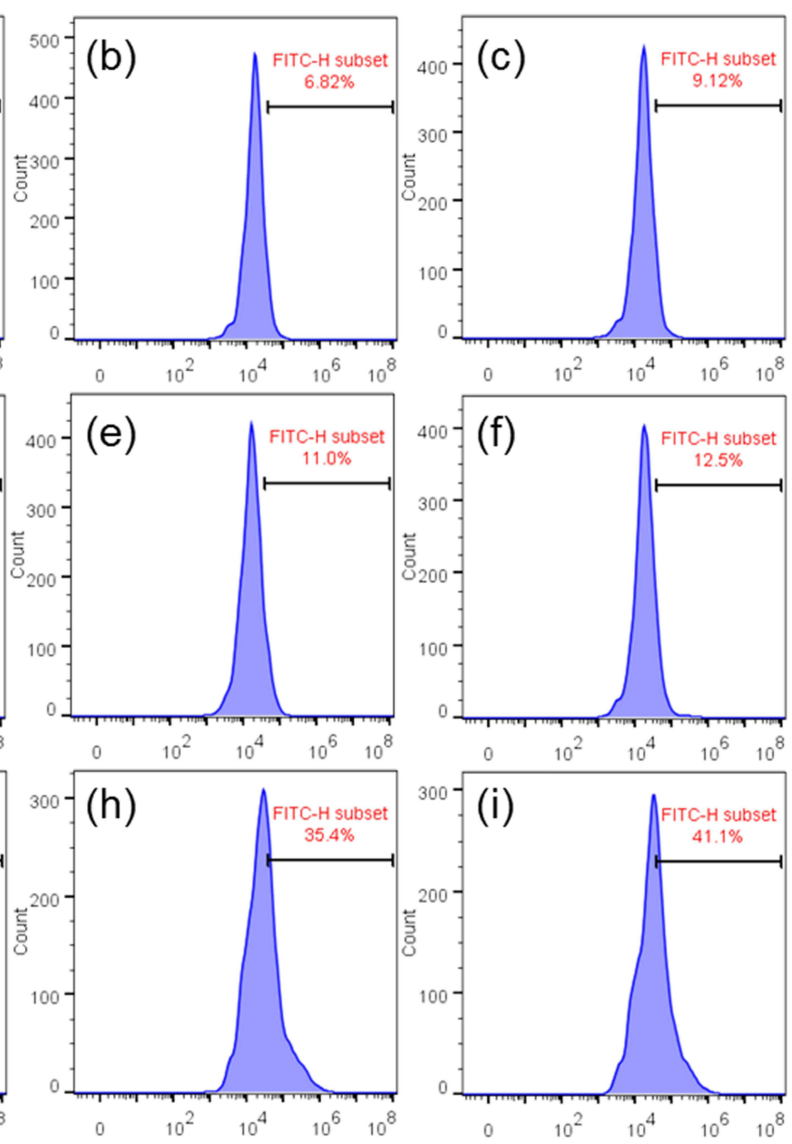

Figure 7 (A) Cellular internalized amounts of PCCNs under different concentrations and time incubation measured by ICP-MS. (B) Intracellular GSH depletion under different concentrations of PCCNs. (C) Intracellular ROS content was analyzed by flow cytometry after various treatments using DCF as a ROS sensor. (a) represents control group; (b) represents DCF group; (c) represents 660-nm group; (d) represents 1064-nm group; (e) represents PCCNs group; (f) represents $\mathrm{H}_{2} \mathrm{O}_{2}$ group; (g) represents PCCNs plus $\mathrm{H}_{2} \mathrm{O}_{2}$ group; (h) represents $\mathrm{PCCNs}$ plus $\mathrm{H}_{2} \mathrm{O}_{2}$ plus 660 -nm group; (i) represents $\mathrm{PCCNs}$ plus $\mathrm{H}_{2} \mathrm{O}_{2}$ plus 660 -nm plus I064-nm group. (D) Quantitative analysis of the fluorescence intensity of intracellular ROS. The differences among groups were calculated using Student's $t$-test. Data are presented as the mean \pm standard error of the mean. ${ }^{* *} \mathrm{p}<0.00 \mathrm{I}$.

\section{Intracellular GSH-Depletion and ROS-Generated Properties}

We next tested the intracellular GSH-depletion property of PCCNs using the GSH assay kit. As shown in Figure 7B, the intracellular GSH-consumption gradually increased as concentration of PCCNs increasing, which destroyed cellular antioxidant defense system and amplified oxidative stress in TME. To further demonstrate ROS generation in 4T1 cells, a fluorescent probe DCF was used to detect the intracellular ROS production. Compared with that in the groups of DCF, $\mathrm{H}_{2} \mathrm{O}_{2}, 660 \mathrm{~nm}, 1064 \mathrm{~nm}$ and PCCNs, an obvious green fluorescence intensity was observed in $4 \mathrm{~T} 1$ cells with PCCNs $/ \mathrm{H}_{2} \mathrm{O}_{2}$ or $\mathrm{PCCNs} / \mathrm{H}_{2} \mathrm{O}_{2}$ plus $660 \mathrm{~nm}$ treatment (Figure 7C), suggesting that PCCNs could increase ROS generation through CTT and PDT effect. Moreover, the 4T1 cells treated with PCCNs plus $\mathrm{H}_{2} \mathrm{O}_{2}$ under the 660 and 1064- $\mathrm{nm}$ laser simultaneously irradiation displayed significantly stronger fluorescence intensity than those under $\mathrm{PCCNs} / \mathrm{H}_{2} \mathrm{O}_{2}$ or $\mathrm{PCCNs} / \mathrm{H}_{2} \mathrm{O}_{2}$ plus $660 \mathrm{~nm}$ (Figure 7D). This indicated that the PTT effect of PCCNs increased ROSenhanced capacity. Taken together, these results showed that the multifunctional PCCNs could display the multipleamplifying ROS effect based on PDT, CTT, PTT-enhanced CTT/PDT and TME-modulated properties.

\section{In vitro Anticancer Effect of PCCNs}

A standard CCK-8 assay was then applied to evaluate the cytotoxicity of PCCNs with different concentrations $(0$, $7.5,15,30,60,120$ and $240 \mu \mathrm{g} \mathrm{mL}^{-1}$ ) toward noncancerous human umbilical vein endothelial cells (HUVECs) and 4T1 cells. Compared to that toward HUVECs, higher cytotoxicity of PCCNs toward 4T1 cells was observed 
A
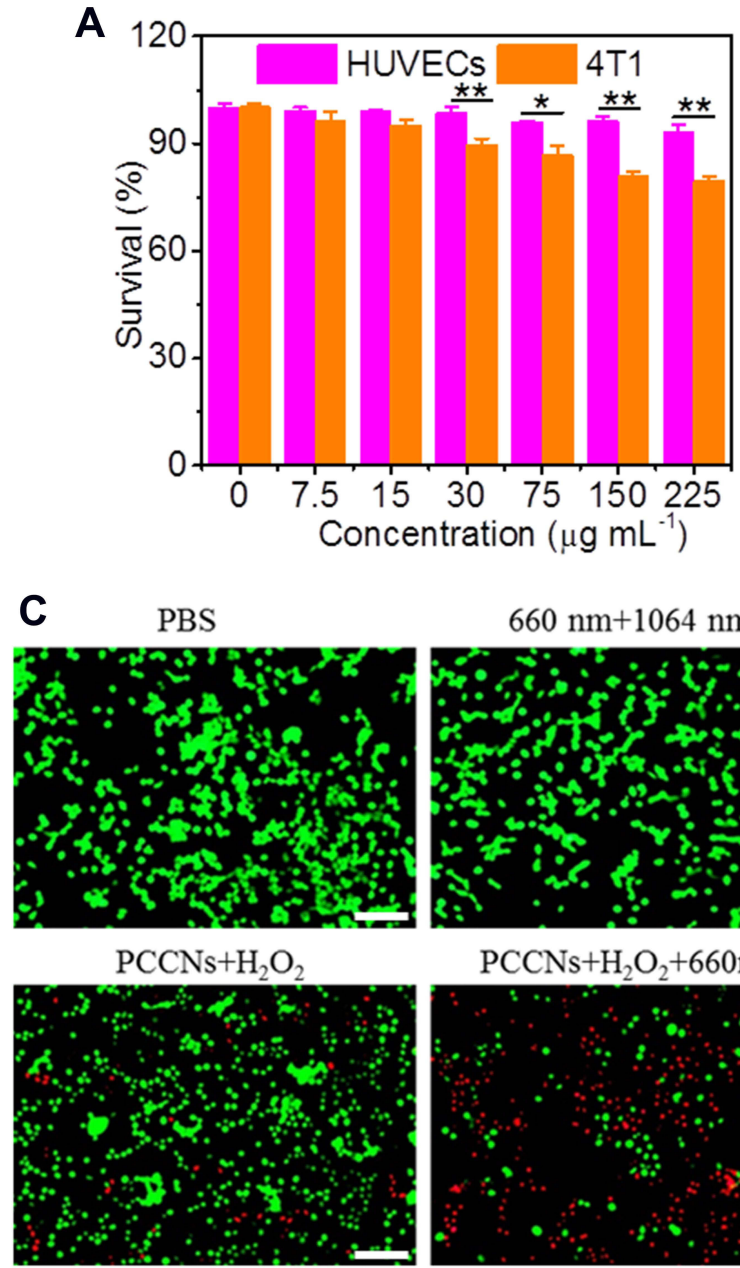

$660 \mathrm{~nm}+1064 \mathrm{~nm}$

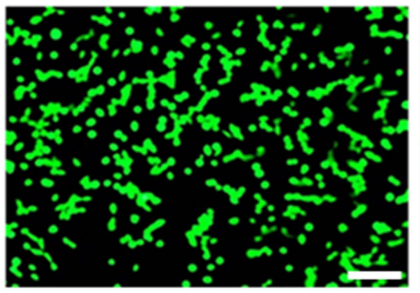

$\mathrm{PCCNs}+\mathrm{H}_{2} \mathrm{O}_{2}+660 \mathrm{~nm}$

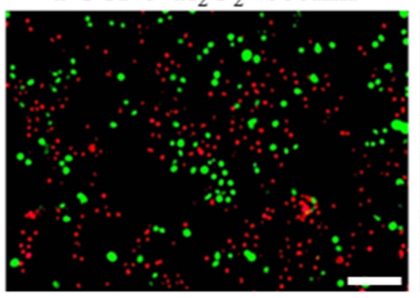

B

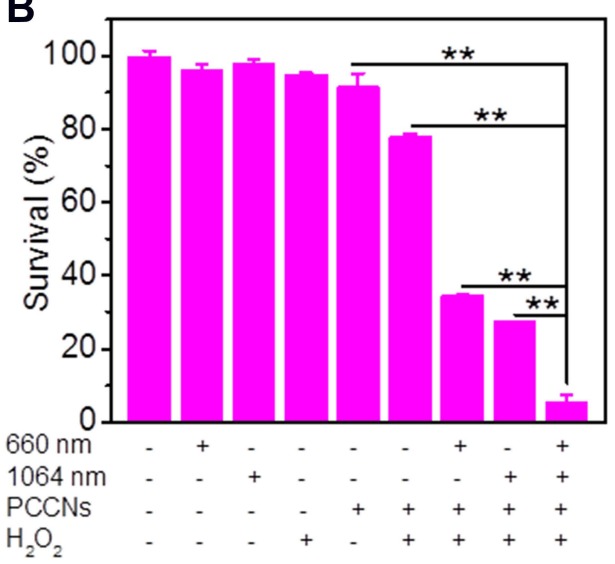

PCCNs
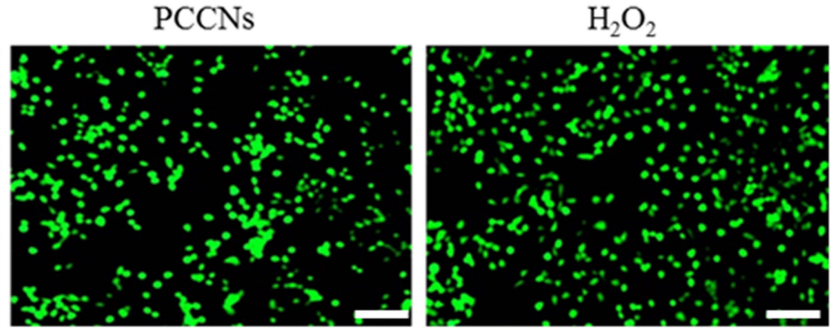

$\mathrm{PCCNs}+\mathrm{H}_{2} \mathrm{O}_{2}+1064 \mathrm{~nm}$

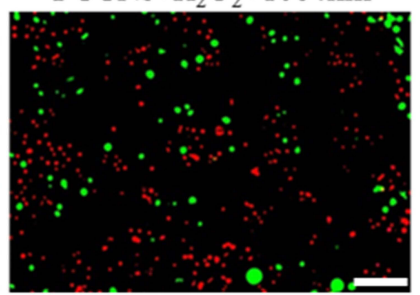

$\mathrm{PCCNs}+\mathrm{H}_{2} \mathrm{O}_{2}+660+1064 \mathrm{~nm}$

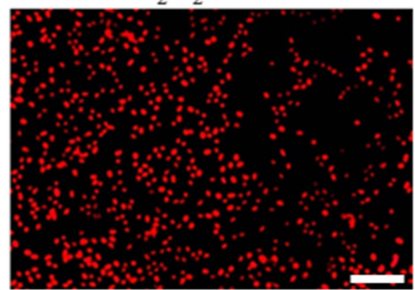

Figure 8 (A) Relative viability of HUVECs and 4TI cells with different concentrations of PCCNs for $24 \mathrm{~h}$. (B) Cell survival rate of the synergistic comprehensive treatment effects of PCCNs by the CCK-8 assay. (C) Fluorescence images of $4 \mathrm{TI}$ cells after various treatments and the cells were co-stained with calcein-AM (green, living cells) and propidium iodide (red, dead cells). Scale bars represent $100 \mu \mathrm{m}$. The differences among groups were calculated using Student's $t$-test. Data are presented as the mean \pm standard error of the mean. ${ }^{*} p<0.05,{ }^{*} p<0.01$.

(Figure 8A), suggesting that PCCNs displayed good biocompatibility and high cytotoxicity toward normal cells and cancer cells, respectively. The significant differences may be attributed to generating more $\cdot \mathrm{OH}$ of PCCNs via catalytic reactions in cancer cells with high $\mathrm{H}_{2} \mathrm{O}_{2}$ concentrations. The effect of the cellular uptake time has been investigated. No obvious cytotoxicity of the PCCNs toward HUVECs was found during $48 \mathrm{~h}$ incubation (Figure S8). The results showed that the PCCNs induced the stronger cell death with the increasing time. Next, to investigate the anticancer effect of PCCNs, a CCK-8 assay was used to evaluate their cytotoxicity in $4 \mathrm{~T} 1$ cells exposed to different treatments. No obvious cell cytotoxicity was found in 4T1 cells with only PBS, 660 plus 1064 $\mathrm{nm}, \mathrm{PCCNs}$, and $\mathrm{H}_{2} \mathrm{O}_{2}$ treatment, while the cell viability was significantly decreased with $\mathrm{PCCNs} / \mathrm{H}_{2} \mathrm{O}_{2}$ and plus 660 or $1064 \mathrm{~nm}$ (Figure $8 \mathrm{~B}$ ). The results demonstrated the feasibility of PCCNs to cause partial cell death through CTT or PDT or PTT alone. Moreover, over $95 \%$ of the cell death was observed in $4 \mathrm{~T} 1$ cells treated with $\mathrm{PCCNs} / \mathrm{H}_{2} \mathrm{O}_{2}$ plus 660- and 1064-nm laser irradiation. This indicated that PCCNs could act a synergistical effect in cancer therapies. In calcein-AM and PI staining (Figure 8C), no red fluorescence was observed in 4T1 cells with PBS, 660 plus $1064 \mathrm{~nm}$, PCCNs, and $\mathrm{H}_{2} \mathrm{O}_{2}$ treatment, suggesting that they cannot induce cell cytotoxicity. In contrast, the highest red fluorescence was found in $4 \mathrm{~T} 1$ cells treated with PCCNs $/ \mathrm{H}_{2} \mathrm{O}_{2}$ plus 660- and 1064-nm laser irradiation compared with $\mathrm{PCCNs} / \mathrm{H}_{2} \mathrm{O}_{2}$ and $\mathrm{PCCNs} / \mathrm{H}_{2} \mathrm{O}_{2}$ plus 660 or $1064 \mathrm{~nm}$. Therefore, these results confirmed the 
excellent anti-cancer efficacy of PCCNs via a synergistical PDT, CTT, PTT-enhanced CTT/PDT and TME-modulated effect.

\section{In vivo Tumor Dual-Modal Imaging}

To explore the potential capability of PCCNs for photoacoustic imaging (PAI), in vitro reconstructed PAI was performed. The PAI intensity of PCCNs significantly increased when their concentration ranged from 25 to $200 \mu \mathrm{g} \mathrm{mL}^{-1}$, and displayed linear relationship $\left(\mathrm{R}^{2}=0.994\right)$ with the concentration (Figure S9). Next, PAI in 4T1 tumor-bearing mice was performed to monitor the signals from a cross section of the tumor at different time intervals after injection. As shown in Figure 9A, the PAI signal intensity reached a peak at 6 $\mathrm{h}$ after injection and exhibited over three times higher than those at $0 \mathrm{~h}$ (Figure 9B), and then began to decrease by 24 h. These results demonstrated that PCCNs could be used for PAI imaging in vivo. To validate the PTT effect of PCCNs in vivo, mice tumors were irradiated using a 1064-nm laser $\left(0.9 \mathrm{~W} \mathrm{~cm}^{-2}, 6 \mathrm{~min}\right)$. PTT imaging showed that the temperature of the tumor treated with PCCNs gradually increased with increasing irradiation time (Figure 9C) and increased to $49.86^{\circ} \mathrm{C}$ while the temperature of the PBS treatment was not remarkably elevated (Figure 9D). The results demonstrated that PCCNs could be powerful PTT agents to ablate tumors under laser irradiation.

A
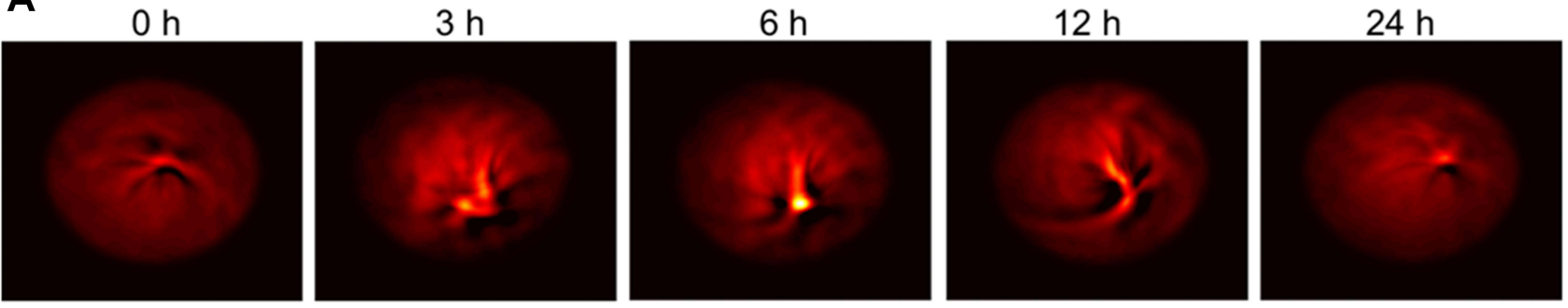

Low

High

B

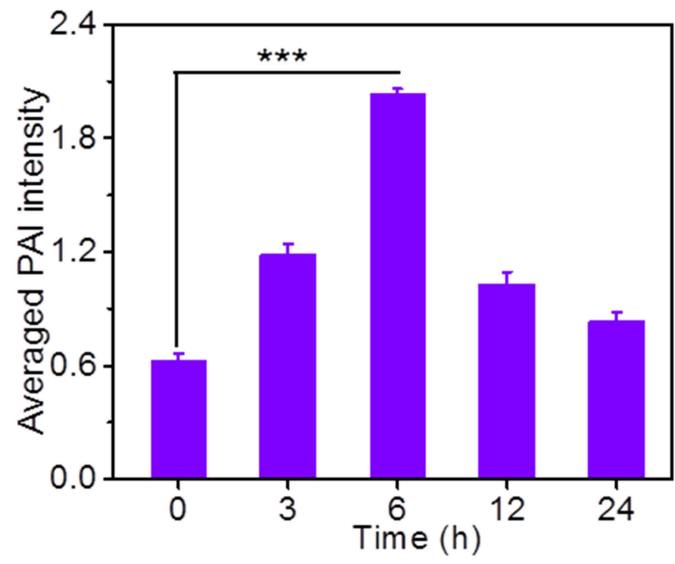

C

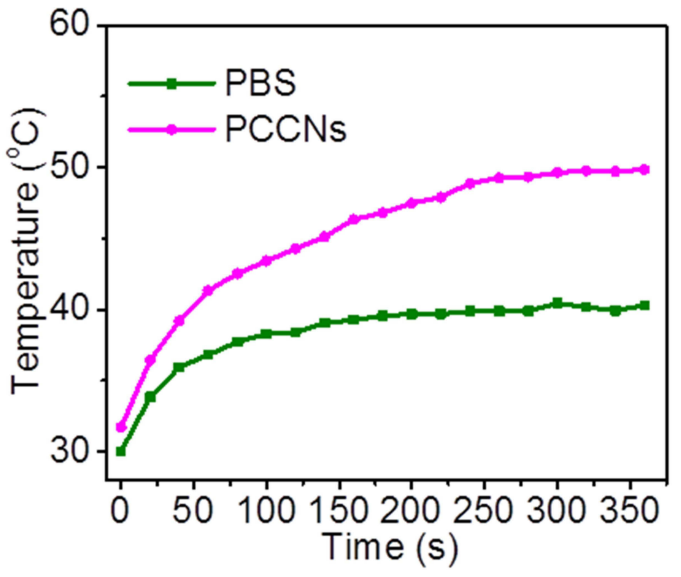

D

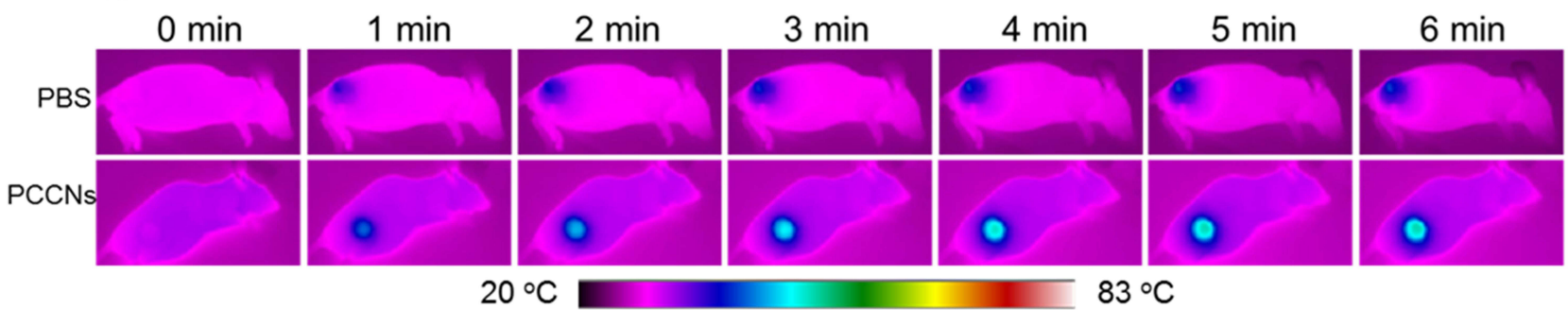

Figure 9 In vivo tumor dual-modal imaging. Photoacoustic imaging (A) and averaged PAl intensity (B) of the tumor-bearing mce intravenously injected with PCCNs at 0,3 , 6, 12, and $24 \mathrm{~h}$, respectively. The PTT images (C) and temperature elevation curves (D) of tumor-bearing mice measured after intravenous injection of PBS and PCCNs plus further laser irradiation. The differences among groups were calculated using Student's $t$-test. Data are presented as the mean \pm standard error of the mean. *** $\mathrm{P}<0.00 \mathrm{I}$. 


\section{In vivo Biodistribution}

To evaluate the in vivo biodistribution, the $\mathrm{Cu}$ content in the major organs at different times after injection was measured by ICP-MS. As shown in Figure S10, the $\mathrm{Cu}$ content was very low in the heart, spleen, and lung at all-time points. A high level of $\mathrm{Cu}$ was observed in the liver because the liver is the main organ of metabolism. $\mathrm{Cu}$ was found in the kidney after injection, indicating rapid $\mathrm{Cu}$ clearance by the renal-urinary system. Importantly, the high accumulation of PCCNs in the tumor site was demonstrated because their small size favored the enhanced permeability and retention (EPR) effect. The high biodistribution of PCCNs in tumor tissues further showcased the in vivo ROS-involved curative effect.

\section{In vivo Synergistic Therapy}

Motivated by the effective accumulation of PCCNs in the tumors under the guidance of PAI and PTT imaging, the synergistic antitumor efficiency in vivo was evaluated. The xenograft 4T1 tumor models were established and tumorbearing mice were randomly divided into five groups: 1) PBS, 2) PCCNs (CTT group), 3) PCCNs plus 660-nm laser (CTT/PDT group), 4) PCCNs plus 1064-nm laser (CTT/PTT group), 5) PCCNs plus 660-nm and 1064-nm laser (CTT/PDT/PTT group). Compared with PBS, CTT group could partially induce tumor growth suppression (Figure 10A), high inhibition rate (Figure S11), and low tumor weight (Figure S12) and size (Figure 10B). In contrast, the CTT/PDT and CTT/PTT group caused the obvious tumor growth suppression, higher inhibition rate, and lower tumor weight and size. Moreover, the CTT/ PDT/PTT group could almost ablate the tumor compared to other groups. In addition, the longer survival rates of mice in CTT/PDT/PTT group were observed posttreatment after 38 days compared to other groups (Figure 10C). The GSH consumption (Figure 10D) and hypoxia alleviation in HIF-1 $\alpha$ stained tumor slices (Figure 10E) were observed after PCCNs treatment, which indicated that PCCNs could regulate the TME. Furthermore, the CTT/PDT/PTT group could induce the most remarkable tissue damage in tumor H\&E-staining slices among all groups (Figure 10F). TUNEL results showed that the CTT/PDT/PTT group significantly caused tumor cell apoptosis in tumor tissues compared to other groups (Figure 10G). Taken together, these results demonstrated that PCCNs with the property of disrupting ROS homeostasis-mediated therapy could effectively improve the therapeutic effectiveness.

\section{Biosafety Evaluation in vivo}

The long-term safety of PCCNs in vivo is a great concern for further bioapplications. During the treatment process, no obvious changes in mice weight (Figure 11A) in all groups, suggesting the excellent long-term biosafety of PCCNs. As shown in Figure 11B, no obvious tissue damage or inflammatory lesions in the major organs, including heart, liver, spleen, lung, and kidney, were observed in PBS and PCCNs groups, indicating the good biocompatibility of PCCNs. The serum levels of serum aspartate transaminase (AST) and alanine transaminase (ALT) from all treatment groups showed no significant difference, indicating that PCCNs had low liver toxicity (Figure 11C and D). Moreover, no obvious abnormal mouse behaviors were visualized during the treatment process. The above results revealed that PCCNs could display excellent biocompatibility and biosafety in vivo for biomedical applications.

\section{Discussion}

We proposed that the novel approaches of disrupting ROS homeostasis-involved therapy presented here had an outstanding potential for anticancer therapy. Maintaining intracellular ROS homeostasis was essential to drive cell growth and proliferation. ${ }^{3}$ ROS homeostasis was disrupted by augmenting ROS generation and weakening cellular antioxidant defense system, thus causing cancer cells apoptosis or necrosis. ${ }^{4,5}$ PCCNs mediated cascade-amplifying ROS generation through PDT, CTT effect, and PTT-enhanced CTT/ PDT activities. Importantly, PCCNs displayed hypoxiarelieved activity and GSH-consumption capability which impaired the antioxidant defenses of tumor cells to disrupt intracellular ROS homeostasis.

In this study, we designed and prepared a novel nanozyme of PCCNs using a facile one-step hydrothermal method. Because of strong conjugation of $\mathrm{Cu}$ ions with carboxyl groups of $\mathrm{Ce} 6$ and thiol groups of PEG-SH, ${ }^{46,50}$ Ce6 and PEG-SH were not only utilized to be the capping agent for stabilizing PCCNs but also used as template for homogeneous growth of PCCNs to obtain uniform distribution of particle size. Unlike previous methods, ${ }^{42}$ we created a rapid one-step strategy and user-friendly synthesis process that did not require toxic organic solvents and raw materials. Most importantly, PCCNs could be acted as CTT, PDT, PTT, and TME-modulated agents for effective antitumor treatment without further surface modification.

In addition, $\mathrm{Cu}$-based nanozymes have recently been discovered more potential for CTT. Although a large 

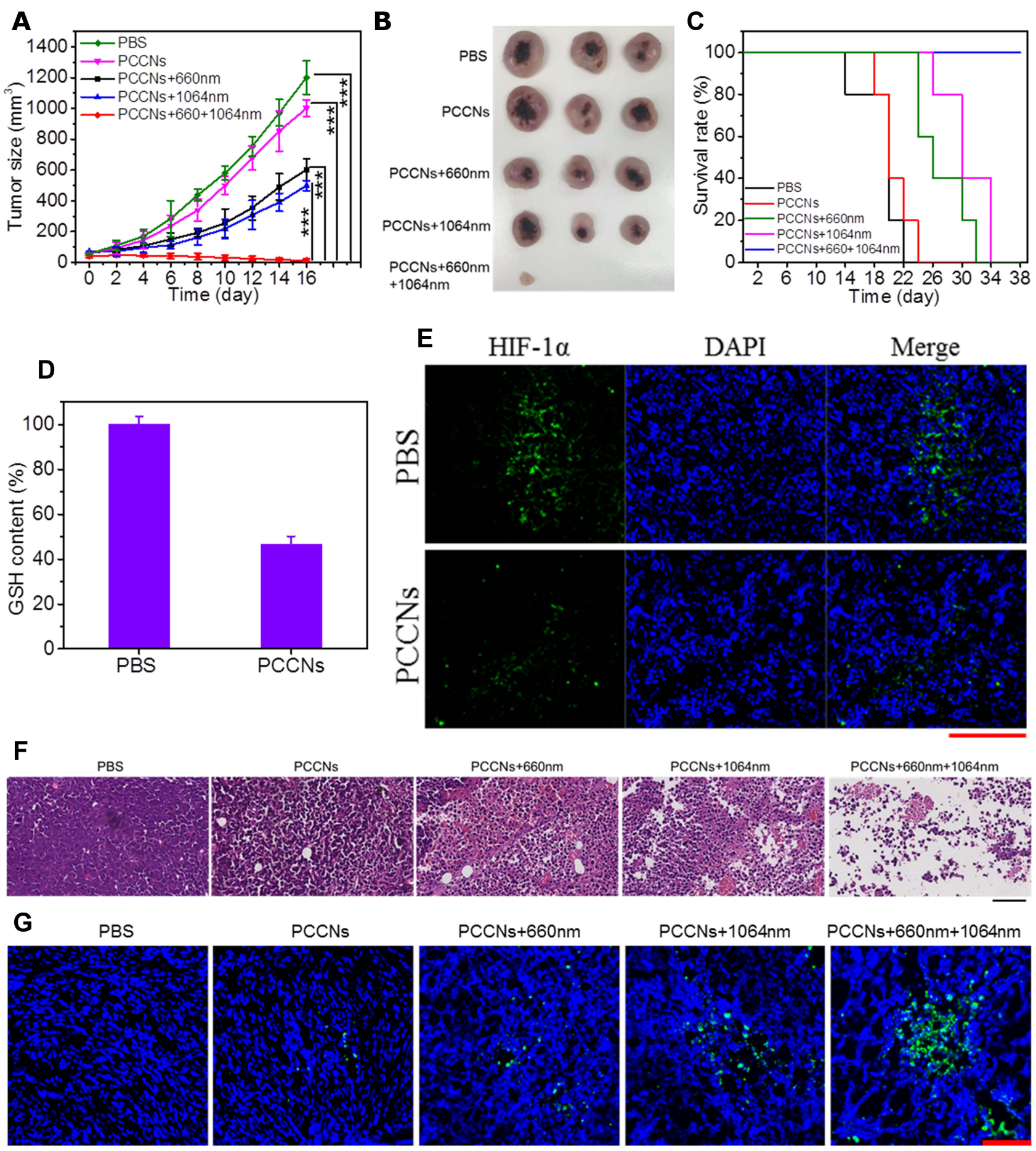

Figure 10 In vivo synergistic therapy. (A) Tumor volume curve of mice treated with (I) PBS, (2) PCCNs, (3) PCCNs plus 660-nm laser, (4) PCCNs plus I064-nm laser, and (5) PCCNs plus 660- plus 1064-nm laser. (B) The digital photographs of tumors from different groups after different treatments. (C) Survival rates of mice after different treatments. The GSH depletion (D) in tumor tissues and HIF-I $\alpha$ (E) stained tumor slides after PCCNs treatment. H\&E (F) and TUNEL (G) staining tumor slides after different treatments. Scale bar: $100 \mu \mathrm{m}$. The differences among groups were calculated using Student's $t$-test. Data are presented as the mean \pm standard error of the mean. $*_{p}<0.01$, **** $\mathrm{p}<0.001$.

number of metal ion-based nanomaterials (eg, Mn, Fe, and Mo) have been discovered more adaptable to act as CTT agents, these materials suffered from the slow catalytic reaction rates under the higher $\mathrm{pH}(6.5-6.9)$ of the
TME. ${ }^{25,26,30} \mathrm{Cu}$ in nanozymes of PCCNs exhibited enzyme-mimic catalytic activity to produce highly toxic - OH for mediating CTT under neutral conditions. Importantly, the PCCNs not only displayed the catalase- 

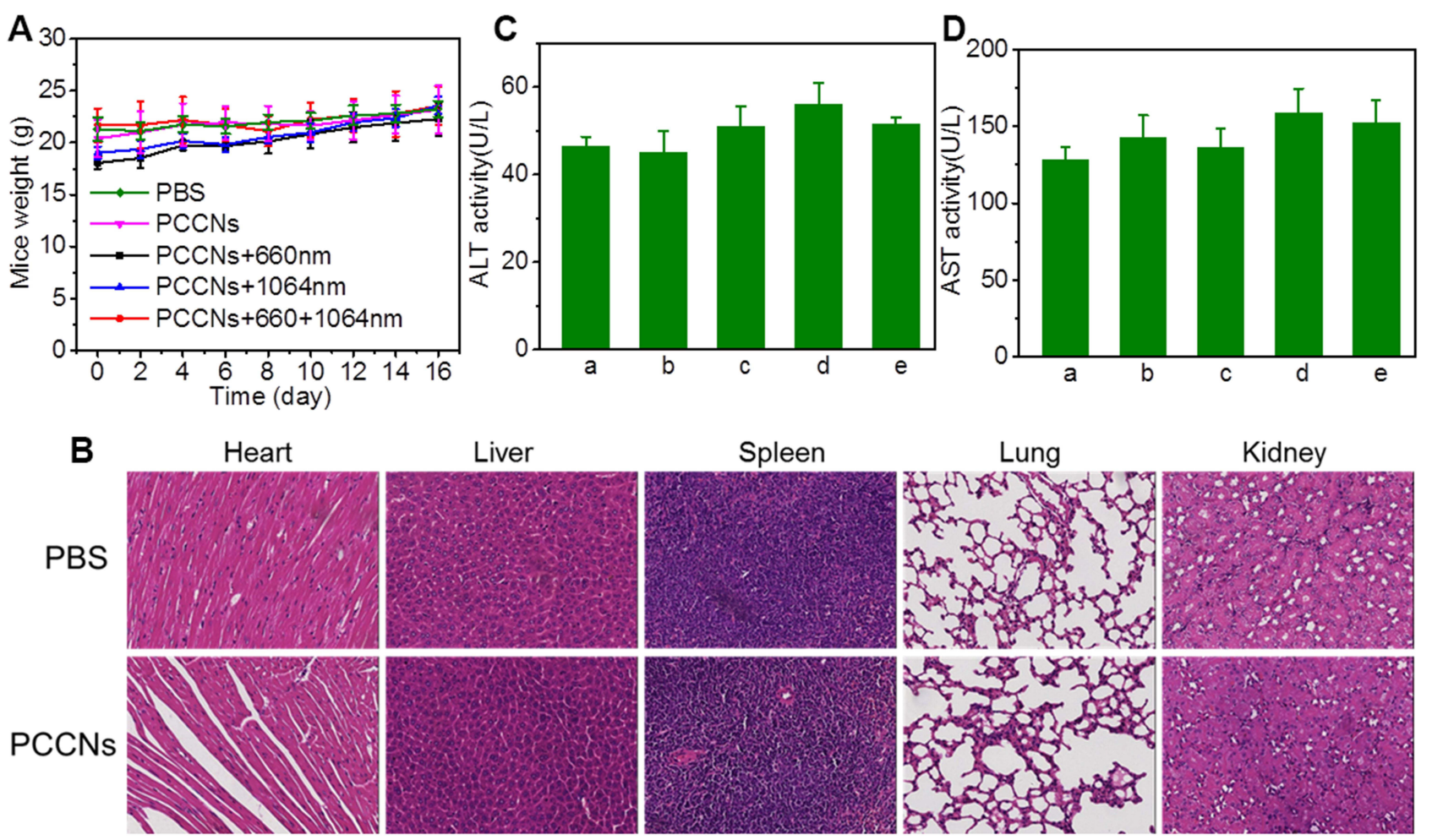

Figure II The in vivo biosafety evaluation. (A) Body weight curve of mice treated with (a) PBS, (b) PCCNs, (c) PCCNs plus 660-nm laser, (d) PCCNs plus I064-nm laser, and (e) PCCNs plus 660- plus 1064-nm laser. (B) H\&E-stained images of heart, liver, spleen, lung, and kidney of the mice treated with PBS and PCCNs. Scale bar stands for $100 \mu \mathrm{m}$. Serum levels of ALT (C) and AST (D) from different groups after different treatments.

like activity to generate $\mathrm{O}_{2}$ in the presence of excess $\mathrm{H}_{2} \mathrm{O}_{2}$, further relieving the $\mathrm{O}_{2}$-dependent limitations of PDT, but also catalyzed intracellular GSH to GSSH for accelerating GSH depletion. This destroyed cellular antioxidant defense system and regulated the TME to disrupt intracellular ROS homeostasis. More importantly, although many nanomaterials have been developed as PTT agents, such as carbon nanomaterials, metal nanomaterials (eg, $\mathrm{Au}$ nanorod, $\mathrm{Ag}$ nanoparticles, Bi nanoparticles, etc.), these materials exhibited the NIR-I absorbance and the low photothermal conversion efficiency. ${ }^{9,25,51}$ The PCCNs showed excellent photothermal performance in the NIRII region and high photothermal conversion efficiency, which was higher than many other nanomaterials. NIR-IIexcited hyperthermia not only could be effectively applied to PTT but also significantly enhanced the CTT/PDT activities to amplify ROS generation. Taken together, PCCNs could act as "all in one" theranostic agents with catalytic, photodynamic/photothermal, and TMEmodulated properties for significantly disrupting ROS homeostasis to favor ROS-mediated therapy.

\section{Conclusions}

In summary, we successfully synthesized multifunctional nanozymes of PCCNs through a facile one-step hydrothermal method. The PCCNs were considered as "all-in-one" nanozymes as they acted to cascade-amplify ROS production and weaken antioxidant defense system to disrupt ROS homeostasis for effective antitumor treatment. PCCNs produced ${ }^{1} \mathrm{O}_{2}$ when exposed to laser irradiation at $660 \mathrm{~nm}$ for PDT. In addition, PCCNs exhibited enzyme-mimic catalytic activity to produce highly toxic $\cdot \mathrm{OH}$ to mediate $\mathrm{CTT}$ under neutral conditions. Moreover, TME could be regulated by PCCNs with the property of $\mathrm{O}_{2}$ evolution and GSH consumption, which relieved the hypoxic to achieve enhanced PDT and impaired the antioxidant defenses of tumor cells, respectively. More importantly, PCCNs displayed photothermal hyperthermia in the NIR-II region, which significantly enhanced the CTT/PDT activities to amplify ROS generation. Both in vitro and in vivo experiments demonstrated that the PCCNs could lead to effectively eliminate tumor cells via nanozyme-mediated synergistic therapy. Overall, the concept of NIR-II-triggered hyperthermia-enhanced PDT/PTT/CTT 
therapy of "all-in-one" nanozymes via disrupting ROS homeostasis highlighted a great promise for future biomedical applications.

\section{Abbreviations}

ROS, reactive oxygen species; TME, tumor microenvironment; PEG, polyethylene glycol; Ce6, chlorin e6; PCCNs, PEG@ $\mathrm{Cu}_{2-\mathrm{x}} \mathrm{S} @ \mathrm{Ce} 6$ nanozymes; PDT, photodynamic therapy; CTT, catalytic therapy; GSH, glutathione; GSSG, glutathione disulfide; PTT, photothermal therapy; ${ }^{1} \mathrm{O}_{2}$, singlet oxygen; $\bullet \mathrm{OH}$, hydroxyl radical; $\cdot \mathrm{O}_{2}^{-}$, superoxide; NIR, nearinfrared; TEM, transmission electron microscope; HRTEM, high-resolution TEM; XRD, X-ray diffraction; SAED, selected area electron diffraction; EDS, energy-dispersive spectroscopy; XPS, X-ray photoelectron spectroscopy; FT-IR, Fourier transform infrared spectroscopy; DCF, 2', 7'dichlorofluorescein diacetate; TMB, 3,3',5,5'-tetramet hylbenzidine; ICP-MS, inductively coupled plasma mass spectrometry; calcein-AM, calcein acetoxymethyl ester; PI, propidine iodide; PAI, photoacoustic imaging; EPR, enhanced permeability and retention; AST, aspartate transaminase; ALT, alanine transaminase; DTNB, (5,5'-Dithiobis-(2-nitrobenzoic acid)); DPBF, 1,3-diphenylisobenzofuran; $\mathrm{H}_{2} \mathrm{O}_{2}$, hydrogen peroxide; CCK-8, Cell Counting Kit-8; DLS, dynamic light scattering; 4T1, mouse mammary carcinoma; HUVECs, human umbilical vein endothelial cells; H\&E, hematoxylin and eosin.

\section{Acknowledgments}

This work was supported by the National Key R\&D Program of China (2019YFA0904400), the Science and Technology Development Fund, Macau SAR (File no. FDCT/131/2016/A3, FDCT/0015/2918/A1), the MultiYear Research Grant (MYRG2019-00069-FHS), the Startup Research Grand (SRG2016-00082-FHS), and the Intramural Research Fund of FHS, University of Macau.

\section{Disclosure}

The authors report no conflicts of interest in this work.

\section{References}

1. Ramsey MR, Sharpless NE. ROS as a tumour suppressor? Nat Cell Biol. 2006;8(11):1213-1215. doi:10.1038/ncb1106-1213

2. Fruehauf JP, Meyskens FL. Reactive oxygen species: a breath of life or death? Clin Cancer Res. 2007;13(3):789. doi:10.1158/1078-0432. CCR-06-2082
3. Schumacker PT. Reactive oxygen species in cancer cells: live by the sword, die by the sword. Cancer Cell. 2006;10(3):175-176. doi:10.1016/j.ccr.2006.08.015

4. Glass SB, Gonzalez-Fajardo L, Beringhs AOR, Lu X. Redox potential and ROS-mediated nanomedicines for improving cancer therapy. Antioxid Redox Signal. 2017;30(5):747-761. doi:10.1089/ ars.2017.7370

5. Nan W, Yue W, Jinlei B, et al. Current development of ROS-modulating agents as novel antitumor therapy. Curr Cancer Drug Targets. 2017;17(2):122-136. doi:10.2174/156800961666616 0216125833

6. Liu J, Yang S, Cao B, et al. Targeting B7-H3 via chimeric antigen receptor $\mathrm{T}$ cells and bispecific killer cell engagers augments antitumor response of cytotoxic lymphocytes. J Hematol Oncol. 2021;14 (1):21. doi:10.1186/s13045-020-01024-8

7. Hu D, Sheng Z, Gao G, et al. Activatable albumin-photosensitizer nanoassemblies for triple-modal imaging and thermal-modulated photodynamic therapy of cancer. Biomaterials. 2016;93:10-19. doi:10.1016/j.biomaterials.2016.03.037

8. Zhu L, Liu J, Zhou G, et al. Targeting immune checkpoint B7-H3 antibody-chlorin e6 bioconjugates for spectroscopic photoacoustic imaging and photodynamic therapy. Chem Commun. 2019;55 (95):14255-14258. doi:10.1039/C9CC06839B

9. Yang D, Yang G, Yang P, et al. Assembly of Au plasmonic photothermal agent and iron oxide nanoparticles on ultrathin black phosphorus for targeted photothermal and photodynamic cancer therapy. Adv Funct Mater. 2017;27(18):1700371. doi:10.1002/adfm.201700371

10. Hou X, Tao Y, Li X, et al. CD44-targeting oxygen self-sufficient nanoparticles for enhanced photodynamic therapy against malignant melanoma. Int J Nanomedicine. 2020;15:10401-10416. doi:10.2147/ IJN.S283515

11. Zhou Z, Song J, Nie L, Chen X. Reactive oxygen species generating systems meeting challenges of photodynamic cancer therapy. Chem Soc Rev. 2016;45(23):6597-6626. doi:10.1039/ c6cs00271d

12. Li M, Zhang H, Hou Y, et al. State-of-the-art iron-based nanozymes for biocatalytic tumor therapy. Nanoscale Horiz. 2020;5(2):202-217. doi:10.1039/C9NH00577C

13. Wu C, Wang S, Zhao J, et al. Biodegradable Fe(III)@WS2-PVP nanocapsules for redox reaction and TME-enhanced nanocatalytic, photothermal, and chemotherapy. Adv Funct Mater. 2019;29 (26): 1901722. doi: $10.1002 / \mathrm{adfm} .201901722$

14. Chen Q, Yang D, Yu L, Jing X, Chen Y. Catalytic chemistry of iron-free Fenton nanocatalysts for versatile radical nanotherapeutics. Mater Horiz. 2020;7(2):317-337. doi:10.1039/ C9MH01565E

15. Lin H, Chen Y, Shi J. Nanoparticle-triggered in situ catalytic chemical reactions for tumour-specific therapy. Chem Soc Rev. 2018;47 (6):1938-1958. doi:10.1039/C7CS00471K

16. Huo M, Wang L, Chen Y, Shi J. Tumor-selective catalytic nanomedicine by nanocatalyst delivery. Nat Commun. 2017;8(1):357. doi:10.1038/s41467-017-00424-8

17. Zhang C, Bu W, Ni D, et al. Synthesis of iron nanometallic glasses and their application in cancer therapy by a localized Fenton reaction. Angew Chem Int Ed. 2016;55(6):2101-2106. doi:10.1002/ anie. 201510031

18. Kim J, Cho HR, Jeon H, et al. Continuous O2-evolving MnFe2O4 nanoparticle-anchored mesoporous silica nanoparticles for efficient photodynamic therapy in hypoxic cancer. $J$ Am Chem Soc. 2017;139 (32):10992-10995. doi:10.1021/jacs.7b05559

19. Li X, Liu Y, Fu F, et al. Single NIR laser-activated multifunctional nanoparticles for cascaded photothermal and oxygen-independent photodynamic therapy. Nanomicro Lett. 2019;11(1):68. doi:10.1007/ s40820-019-0298-5 
20. Liu C, Wang D, Zhang S, et al. Biodegradable biomimic copper/ manganese silicate nanospheres for chemodynamic/photodynamic synergistic therapy with simultaneous glutathione depletion and hypoxia relief. ACS Nano. 2019;13(4):4267-4277. doi:10.1021/ acsnano.8b09387

21. Wang P, Liang C, Zhu J, et al. Manganese-based nanoplatform as metal ion-enhanced ROS generator for combined chemodynamic/ photodynamic therapy. ACS Appl Mater Interfaces. 2019;11 (44):41140-41147. doi:10.1021/acsami.9b16617

22. Hu P, Wu T, Fan W, et al. Near infrared-assisted Fenton reaction for tumor-specific and mitochondrial DNA-targeted photochemotherapy. Biomaterials. 2017;141:86-95. doi:10.1016/j. biomaterials.2017.06.035

23. Ma B, Wang S, Liu F, et al. Self-assembled copper-amino acid nanoparticles for in situ glutathione "AND" $\mathrm{H} 2 \mathrm{O} 2$ sequentially triggered chemodynamic therapy. $J$ Am Chem Soc. 2019;141 (2):849-857. doi:10.1021/jacs.8b08714

24. Lin L-S, Song J, Song L, et al. Simultaneous Fenton-like ion delivery and glutathione depletion by $\mathrm{MnO}$-based nanoagent to enhance chemodynamic therapy. Angew Chem Int Ed. 2018;57 (18):4902-4906. doi:10.1002/anie.201712027

25. Soltani T, Lee B-K. Enhanced formation of sulfate radicals by metal-doped $\mathrm{BiFeO} 3$ under visible light for improving photo-Fenton catalytic degradation of 2-chlorophenol. Chem Eng J. 2017;313:1258-1268. doi:10.1016/j.cej.2016.11.016

26. Ding B, Shao S, Jiang F, et al. MnO2-disguised upconversion hybrid nanocomposite: an ideal architecture for tumor microenvironment-triggered UCL/MR bioimaging and enhanced chemodynamic therapy. Chem Mater. 2019;31(7):2651-2660. doi:10.1021/ acs.chemmater.9b00893

27. Lin L-S, Huang T, Song J, et al. Synthesis of copper peroxide nanodots for $\mathrm{H} 2 \mathrm{O} 2$ self-supplying chemodynamic therapy. $J \mathrm{Am}$ Chem Soc. 2019;141(25):9937-9945. doi:10.1021/jacs.9b0 3457

28. Liu X, Zhu X, Qi X, Meng X, Xu K. Co-administration of iRGD with sorafenib-loaded iron-based metal-organic framework as a targeted ferroptosis agent for liver cancer therapy. Int $J$ Nanomedicine. 2021;16:1037-1050. doi:10.2147/IJN.S292528

29. Wang Z, Liu B, Sun Q, et al. Fusiform-like copper(II)-based metalorganic framework through relief hypoxia and GSH-depletion coenhanced starvation and chemodynamic synergetic cancer therapy. ACS Appl Mater Interfaces. 2020;12(15):17254-17267. doi:10.1021/ acsami.0c01539

30. Chang $M$, Wang $M$, Wang $M$, et al. A multifunctional cascade bioreactor based on hollow-structured $\mathrm{Cu} 2 \mathrm{MoS} 4$ for synergetic cancer chemo-dynamic therapy/starvation therapy/phototherapy/immunotherapy with remarkably enhanced efficacy. Adv Mater. 2019;31 (51):1905271. doi:10.1002/adma.201905271

31. Chen S-C, Lin C-Y, Cheng T-L, Tseng W-L. 6-mercaptopurineinduced fluorescence quenching of monolayer MoS2 nanodots: applications to glutathione sensing, cellular imaging, and glutathione-stimulated drug delivery. Adv Funct Mater. 2017;27 (41):1702452. doi:10.1002/adfm.201702452

32. Feng W, Han X, Wang R, et al. Nanocatalysts-augmented and photothermal-enhanced tumor-specific sequential nanocatalytic therapy in both NIR-I and NIR-II biowindows. Adv Mater. 2019;31 (5):1805919. doi:10.1002/adma.201805919

33. Wang S, Yang L, Cho H-Y, et al. Programmed degradation of a hierarchical nanoparticle with redox and light responsivity for self-activated photo-chemical enhanced chemodynamic therapy. Biomaterials. 2019;224:119498. doi:10.1016/j.biomaterials.2019.119498

34. Zhu L, Li P, Gao D, et al. pH-sensitive loaded retinal/indocyanine green micelles as an "all-in-one" theranostic agent for multi-modal imaging in vivo guided cellular senescence-photothermal synergistic therapy. Chem Commun. 2019;55(44):6209-6212. doi:10.1039/ C9CC02567G
35. Huang X, Deng G, Han Y, et al. Right Cu2-xS@MnS core-shell nanoparticles as a photo/H2O2-responsive platform for effective cancer theranostics. Adv Sci. 2019;6(20):1901461. doi:10.1002/ advs.201901461

36. Fang X, Lui KH, Li S, et al. Multifunctional nanotheranostic gold nanocage/selenium core-shell for PAI-guided chemo-photothermal synergistic therapy in vivo. Int $J$ Nanomedicine. 2020;15:10271-10284. doi:10.2147/IJN.S275846

37. Zhu L, Gao D, Xie L, Dai Y, Zhao Q. NIR II-excited and pH-responsive ultrasmall nanoplatform for deep optical tissue and drug delivery penetration and effective cancer chemophototherapy. Mol Pharm. 2020;17 (10):3720-3729. doi:10.1021/acs.molpharmaceut.0c00404

38. Han X, Huang J, Jing X, et al. Oxygen-deficient black titania for synergistic/enhanced sonodynamic and photoinduced cancer therapy at near infrared-II biowindow. ACS Nano. 2018;12(5):4545-4555. doi:10.1021/acsnano.8b00899

39. Zhu P, Gao S, Lin H, et al. Inorganic nanoshell-stabilized liquid metal for targeted photonanomedicine in NIR-II biowindow. Nano Lett. 2019;19(3):2128-2137. doi:10.1021/acs.nanolett.9b00364

40. Fan K, Xi J, Fan L, et al. In vivo guiding nitrogen-doped carbon nanozyme for tumor catalytic therapy. Nat Commun. 2018;9(1):1440. doi:10.1038/s41467-018-03903-8

41. Shi W, Wang Q, Long Y, et al. Carbon nanodots as peroxidase mimetics and their applications to glucose detection. Chem Commun. 2011;47(23):6695-6697. doi:10.1039/C1CC11943E

42. Bharathiraja S, Manivasagan P, Moorthy MS, Bui NQ, Lee KD, Oh J. Chlorin e6 conjugated copper sulfide nanoparticles for photodynamic combined photothermal therapy. Photodiagnosis Photodyn Ther. 2017;19:128-134. doi:10.1016/j.pdpdt.2017.04.005

43. Ansari Z, Saha A, Singha SS, Sen K. Phytomediated generation of $\mathrm{Ag}, \mathrm{CuO}$ and $\mathrm{Ag}-\mathrm{Cu}$ nanoparticles for dimethoate sensing. J Photochem Photobiol A. 2018;367:200-211. doi:10.1016/j. jphotochem.2018.08.026

44. Ansari Z, Singha SS, Saha A, Sen K. Hassle free synthesis of nanodimensional $\mathrm{Ni}, \mathrm{Cu}$ and $\mathrm{Zn}$ sulfides for spectral sensing of $\mathrm{Hg}$, $\mathrm{Cd}$ and $\mathrm{Pb}$ : a comparative study. Spectrochim Acta A Mol Biomol Spectrosc. 2017;176:67-78. doi:10.1016/j.saa.2017.01.005

45. Agoro MA, Meyer EL, Mbese JZ, Manu K. Electrochemical fingerprint of CuS-hexagonal chemistry from (Bis(N-1,4-Phenyl$\mathrm{N}$-(4-morpholinedithiocarbamato) copper(II) complexes) as photon absorber in quantum-dot/dye-sensitised solar cells. Catalysts. 2020;10(3):300. doi:10.3390/catal10030300

46. Wang X, Zhang Q, Zou L, Hu H, Zhang M, Dai J. Facile-synthesized ultrasmall $\mathrm{CuS}$ nanocrystals as drug nanocarriers for highly effective chemo-photothermal combination therapy of cancer. RSC $A d v$. 2016;6(25):20949-20960. doi:10.1039/C5RA23652E

47. Li Q-L, Sun Y, Ren L, et al. Supramolecular nanosystem based on pillararene-capped $\mathrm{CuS}$ nanoparticles for targeted chemo-photothermal therapy. ACS Appl Mater Interfaces. 2018;10 (35):29314-29324. doi:10.1021/acsami.8b09330

48. Liang G, Jin X, Qin H, Xing D. Glutathione-capped, renal-clearable $\mathrm{CuS}$ nanodots for photoacoustic imaging and photothermal therapy. J Mater Chem B. 2017;5(31):6366-6375. doi:10.1039/C7TB01517H

49. Tian Q, Jiang F, Zou R, et al. Hydrophilic Cu9S5 nanocrystals: a photothermal agent with a $25.7 \%$ heat conversion efficiency for photothermal ablation of cancer cells in vivo. ACS Nano. 2011;5 (12):9761-9771. doi:10.1021/nn203293t

50. Ding K, Zeng J, Jing L, et al. Aqueous synthesis of PEGylated copper sulfide nanoparticles for photoacoustic imaging of tumors. Nanoscale. 2015;7(25):11075-11081. doi:10.1039/C5NR02180D

51. Xie L, Wang G, Zhou H, et al. Functional long circulating single walled carbon nanotubes for fluorescent/photoacoustic imaging-guided enhanced phototherapy. Biomaterials. 2016;103:219-228. doi:10.1016/j. biomaterials.2016.06.058 


\section{Publish your work in this journal}

The International Journal of Nanomedicine is an international, peerreviewed journal focusing on the application of nanotechnology in diagnostics, therapeutics, and drug delivery systems throughout the biomedical field. This journal is indexed on PubMed Central, MedLine, CAS, SciSearch ${ }^{\mathbb{B}}$, Current Contents ${ }^{\mathbb{B}} /$ Clinical Medicine,
Journal Citation Reports/Science Edition, EMBase, Scopus and the Elsevier Bibliographic databases. The manuscript management system is completely online and includes a very quick and fair peer-review system, which is all easy to use. Visit http://www.dovepress.com/ testimonials.php to read real quotes from published authors.

Submit your manuscript here: https://www.dovepress.com/international-journal-of-nanomedicine-journal 